\title{
THE CONTROL OF AN ELASTIC FLUID
}

\author{
H. BATEMAN
}

\section{INTRODUCTION}

1.1. Introduction. Mathematicians should pause periodically in their own work and peruse the progress in astronomy, biology, chemistry, economics, engineering, and physics to see if recent advances in these fields suggest problems of mathematical interest. One reason why the Gibbs Lectureship was founded was, indeed, to facilitate a fruitful friendliness between mathematicians and other scientists.

The subject of control is now very important and promises to be so in the future. Much has been written about the control of the air, the control of ships, airplanes, balloons, bombs, gliders, robots and torpedoes. The regulation of rotation became important in the early days of the telescope and steam engine. The related problem of stability is important now for electric motors, marine engines, hydraulic turbines and the generating plants for the distribution of gas and electricity for there is generally an economical speed of operation. In radio telegraphy a certain speed may be needed in order to get a desired frequency.

Controls are necessary in the chemical industries and in mining. They are useful in entertainment and were much needed when arc lights were used for illumination. Fountains which begin to play automatically at sunset are used at exhibitions. Appold's home in London had many automatic devices to interest visitors.

The control of conditions under which observations are made is of great importance to the astronomer, the physicist and the aeronautical engineer. The designer of an engine plans to regulate the flow, pressure, temperature and composition of his working fluid so that the engine will run smoothly and economically.

The control of combustion may be important not only for economical reasons but also to avoid the production of smoke. On the other hand this production may be desirable sometimes when a smoke screen is needed. In such a case there should be flexibility of control. The subject of control is important also in refrigeration, air conditioning and the preparation of food. Great attention is being paid to human comfort. We are in an era of air conditioning on a large scale

The seventeenth Josiah Willard Gibbs lecture delivered at Chicago, Illinois, November 26, 1943, under the auspices of the American Mathematical Society; received by the editors April 2, 1945. 
and this requires the solution of many problems of control. It is now understood throughout the land that the provision of the proper atmospheric conditions for the comfort of workmen and the performance of good work is even more important than the regulation of the supply of air and fuel to an engine. Precise weather is needed for precision work and for the manufacture of instruments of precision such as gauges. Proper air conditioning is needed for the production of quality fabrics. The proper temperature must be maintained when stained glass windows are being made. In small arms munition works where dry explosives are handled there is inevitably a certain amount of dust and for safety the amount must be regulated. A gas company must regulate the pressure of gas which it distributes and must also regulate the composition so that an escape of gas may be readily detected by the odour of the escaping gas. Controls are needed for the safety of miners and of workmen in many industries. In the purification of drinking water the rate of supply of chlorine must be regulated.

The subject of control is clearly an enormous one and it is well to bear in mind that advances made in one branch of the subject are sometimes useful in another. A recent aerodynamical torque transmitter for the regulation of a marine engine ${ }^{1}$ is based on a principle used in the Remarex carbon dioxide recorder in which there are two pairs of vaned discs, one pair running in air and the other in the flue gas to be tested for $\mathrm{CO}_{2}$ content. The torque transmitter is intended to prevent the marine engine from racing when, owing to the pitching of the ship, the propeller leaves the water.

Thus advances in marine and aeronautical engineering may depend on advances in chemical engineering. They may depend also on advances in electrical engineering. In wind tunnel research it is important to be able to regulate the velocity of the air moving through the tunnel and one way of doing this has been provided by the extensive work on amplidynes made by the General Electric Company. A large adjustable-speed wind tunnel drive based on the use of amplidynes is described in a paper by Clymer. ${ }^{2}$

In acoustical research it is often necessary to control the vibrations of air in a room. In reverberation work, for instance, the generator of

${ }^{1}$ Aerodynamic marine-engine governor, Engineering vol. 157 (1944) pp. 447-448.

2 C. C. Clymer, Large adjustable-speed wind-tunnel drive, Transactions of the American Institute of Electrical Engineers vol. 61 (1942) pp. 156-158. Many applications of the amplidyne in closed cycle controllers or regulating systems are described by F. E. Crever, Fundamental principles of amplidyne applications, ibid. vol. 62 (1943) pp. 603-606. 
sound may be required to produce a pure tone for a certain length of time. If a loud speaker is used and the drive is furnished by an electric current the frequency must be controlled. Sometimes a pure tone is obtained electrically with the aid of an electrical filter. The theory of acoustic and electric filters belongs to the larger subject of the control of vibrations which is important also as it is desirable to eliminate as far as possible the noise and unpleasant vibrations associated with the use of machinery. Much attention has been paid in recent years to the problem of the muffler, the damping of the torsional vibrations of crankshafts and the avoidance of dangerous oscillations in hydraulic transmission lines.

\subsection{Quantities which it may be desirable to control. ${ }^{3}$}

1. The temperature $T$.

2. The total density, $\rho$, or its reciprocal the specific volume.

3 . The speed $q$ or the component velocities $u, v, w$.

4. The mass flow per unit area $q$.

5. The pressure $p$.

6. The heat content or enthalpy $H$.

7. The rate of chemical action, evaporation or condensation.

8. The coefficient of heat transfer.

\footnotetext{
${ }^{3}$ For the control of temperature reference may be made to E. Griffiths, Thermostats and temperature-regulating instruments, Griffin, London, 1943; Th. J. Rhodes, Industrial instruments for measurement and control, McGraw-Hill, New York and London, 1941; R. L. Weber, Temperature measurement and control, Blakiston, Philadelphia, 1941. For the control of various physical quantities see M. Jakob, P. Gmelin and J. Kronert, Physikalische Kontrolle und Regulierung des Betriebes, Part I, Leipzig, 1932, Lithoprint, Edwards Brothers, Ann Arbor, Mich., 1943. For matters relating to heat transfer and evaporation see W. H. McAdams, Heat transmission, McGraw-Hill, New York, 1942; also A. Fono and C. H. Fielding's papers in Engineering vol. 149 (1940) pp. 79-82. In the theory of hydrogen cooling a mathematical theory of the shaft sealing system is given by D. S. Snell, The hydrogen-cooled turbine, Transactions of the American Institute of Electrical Engineers vol. 59 (1940) pp. 3550. For the recent work on detonation and the physics of flames reference may be made to the book of Bernard Lewis and G. v. Elbe, Combustion, flames and explosion of gases, Cambridge, University Press, 1938, and to their paper, Stability and structure of burner flames, Journal of Chemistry and Physics vol. 11 (1943) pp. 75-97. For problems of control in the chemical industry reference may be made to the article by $\mathrm{H}$. Seiferheld, Die Regeltechnik in der chemischen Grossindustrie, Zeitschrift für Technische Physik, vol. 18 (1937) p. 409 and to a paper by M. Ruhemann, Equilibrium of liquid and vapour in a rectifying pan, Physica vol. 4 (1937) pp. 1157-1168, in which use is made of some equations given by F. Bošnjakovic. For the electrical precipitation of particles and fumes in gases reference may be made to the work of F. G. Cottrell, Electrical dust and fume precipitation, Bulletin of the American Institute of Mining Engineers vol. 67 (1912) pp. 667-675, discussion, pp. 675-680.
} 
9. The velocity of propagation of ignition, detonation or wave motion.

10. The increase or rate of increase of the entropy.

\subsection{Types of motion which it may be desirable to produce or avoid.}

1. Motions characterized by a certain degree of turbulence such as:

a. Laminar motion in which there is no turbulence.

b. Flow that seems steady but is really turbulent.

c. Tumultuous flow.

d. Swirling flow which is desirable for efficient mixing and good combustion.

e. Flow in which the stream breaks away from the boundary at a selected place.

f. A standard type of turbulence for comparable results in different wind tunnels.

g. Pulsating flow.

h. Flow in which there is a decided rotation about a fixed axis or a mean direction of motion.

2. Motions in which there is a specified relation between $p$ and $\rho$ (barotropic flow) or between $\rho q$ and $q$.

3. Motions accompanied by regular vibrations which may or may not be audible.

a. Regular vibrations may be desirable in experimental work or in some types of engine. Thus in the Kadency engine the intake is timed to occur at the moment when the pressure in the cylinder has fallen below the atmospheric pressure. In the Constantinesco patents regular vibrations in a fluid are used for various types of control in which accurate timing is essential.

b. Both regular and irregular vibrations may be undesirable on account of the noise they produce or because they make a flame unsteady and lead to its extinction or to flash back.

1.4. Methods of control. As the subject of control belongs largely to chemical and electrical engineering only a brief outline of methods can be given here and these will be restricted largely to cases of aerodynamical interest. A few references are given to books from which the reader can obtain information on chemical and electrical methods.

Some of the most important methods of control are:

1. Clever design of fixed boundaries so as to produce desired results with little attention. Thus diffusors, guide vanes and honeycombs may produce the desired type of flow in a wind channel. A

${ }^{4} \mathrm{~S}$. J. Davies, $A n$ analysis of certain characteristics of a Kadency engine, Engineering vol. 149 (1940) pp. 515-517, 557-559, 617-620. 
spoiler may prevent the flow of air over a roof from injuring the roof. Stationary parts of an engine may be designed to produce a type of flow which is compatible with high efficiency. The wings, body and control surfaces of an airplane may be designed so as to provide low drag and good maneuverability.

2. Devices for altering the form of the boundary of a fluid. Of these the valve is the most important and the design of a suitable valve is often one of the chief steps in the development of a new invention. A list has been formed of nearly eighty different kinds of valves. A valve is generally a device for regulating the rate of flow of a fluid but it may also be used to regulate the pressure or composition of a gas in an enclosure. Mathematically it is usually considered in connection with the regulating device but there are some cases in which equations can be set up for the valve alone. In an attempt to elucidate the action of the throttle valve Joule and Thomson (Lord Kelvin) made their famous porous plug experiment which tests the accuracy of the thermodynamical assumption that the internal energy of a gas depends only on its temperature. Thomson's discussion of the experiment brought into prominence the idea of heat content or enthalpy. Valves may be regarded as including adjustable slots in wings ${ }^{5}$ and devices for sucking air from the boundary layer or for blowing air into the boundary layer. ${ }^{6}$ The ports of a bunsen burner are also valves. Another device for altering the form of the boundary of the fluid is the fan or blower which produces a forced draught. A modification of this is the windmill or air turbine. A steam turbine or turbine working with gas, mercury vapor or some combination of fluids is another modification.

3. Devices for altering the thermal or electrical condition of a fluid at a boundary. The supply of heat at a boundary or the absorption of heat at a boundary is a most effective way of controlling the motion of a fluid. There are also many electrical devices by means of which an aerodynamic or hydraulic system may be coupled with an electrical system that is furnished with some means of control which may or may not be automatic.

4. Devices for introducing solid particles or liquid in the form of a spray into the body of the working fluid. Gases in the form of jets may also be introduced as in the blowpipe torch and in furnaces. Overfire air jets have been found to be effective for smoke elimination

${ }^{5}$ F. Handley Page, The Handley-Page wing, Aeronautical Journal vol. 25 (1921) p. 363.

- O. Schrenk, Boundary layer removal by suction, National Advisory Committee on Aeronautics, Technical Memorandum No. 974, 1941. 
as in a recent paper by Engdahl and Holton. ${ }^{7}$ Davis ${ }^{8}$ has applied a theory of turbulent air jets developed by Tollmien to the problem of the furnace. The ignition of gaseous mixtures by hot moving particles has been studied by Silver ${ }^{9}$ and Paterson. ${ }^{10}$ The cooling of gases by sprayed water is one of the methods employed in air conditioning; it has the advantage that the humidity of the air may thereby be controlled at the same time. A history of air conditioning is given by W. H. Carrier. ${ }^{11}$ There are cases in which a supply of heat may lead to large fluctuation in temperature. The stability of a simple thermal device which has been called an "academic oven" has been considered by Turner. ${ }^{12}$ The analysis depends on a transcendental equation involving both exponential and trigonometrical functions. Oscillations in thermal regulators have been considered also by Himmler. ${ }^{13}$

The problem of stability and of the avoidance of large oscillations becomes important whenever the working fluid is coupled with a mechanical or electrical regulating device. The centrifugal governor invented by Huygens ${ }^{14}$ as a possible means of regulating a clock was adapted for windmills and water wheels before it was used by James Watt for the steam engine. A theory of the governor of Huygens has been given by Poor, ${ }^{15}$ the theory of Watt's governor and related devices has an extensive literature beginning, perhaps, with the work of

${ }^{7}$ R. B. Engdahl, Overfire air jets effective for smoke elimination, Heating, Piping and Air Conditioning, September 1943, p. 481.

${ }^{8}$ R. F. Davis, The mechanics of flame and air jets, Engineering vol. 144 (1937) pp. 608-610, 667-668; Proceedings of the Institute of Mechanical Engineers vol. 137 (1938) pp. 11-72.

${ }^{\circ}$ R. S. Silver, The ignition of gaseous mixtures by hot particles, Philosophical Magazine (7) vol. 23 (1936) pp. 633-657.

${ }^{10} \mathrm{~S}$. Paterson, The ignition of inflammable gases by hot moving particles, Philosophical Magazine (7) vol. 28 (1939) pp. 1-23.

${ }^{11}$ W. H. Carrier, Air conditioning, Encyclopedia Brittanica; see also W. H. Carrier, R. E. Cherne and W. A. Grant, Modern air conditioning, heating and ventilation, Chicago, Pitman, 1940; C. O. Mackey, Air conditioning principles, Scranton, Pa., 1941. Lord Kelvin is credited with a proposal for the use of mechanical cooling as a means of improving human comfort. William Appold devised apparatus for control of temperature and humidity. See J. P. Gassiot, On Appold's apparatus for regulating temperature and keeping the air in a building at any desired degree of moisture. Proc. Roy. Soc. London vol. 15 (1867) pp. 144-146.

${ }^{12}$ L. B. Turner, Self-oscillation in a retroacting thermal conductor, Proc. Cambridge Philos. Soc. vol. 32 (1936) pp. 663-675.

${ }^{13} \mathrm{C}$. Himmler, Die Pendelungen bei warmetechnischen Regelvorgängen, Zeitschrift für Technische Physik vol. 11 (1929) pp. 579-584.

${ }^{14}$ C. Huygens, Horologii oscillatorii, Part 5, Paris, 1673; Horologium, 1658.

${ }^{15}$ V. C. Poor, The Huygens governor, Amer. Math. Monthly vol. 32 (1925) pp. 115121. 
Navier and Poncelet. ${ }^{16}$ For a survey of this literature reference may be made to the article of von Mises and to the book of Tolle. ${ }^{17}$

\section{The ALGEBRAIC PROBLEM}

2.1. Conditions of stability. In direct regulation the stability of the dynamical, electrical or hydraulic regulating device often can be discussed by the method of small oscillations. The system is generally a compound one which is partly of one type and partly of another. For instance, in the case of the steam engine, the compound system consists of the steam, the valve and the centrifugal governor and so the processes taking place are described by a system of differential equations. In indirect regulation the system is again compound. When a deviation from the norm (or rated value of the quantity to be controlled) passes out of the region of insensitivity, the indicator actuates a motor through the amplifier and a disturbance is produced which tends to annul the disturbance shown by the indicator. When the transient force is no longer operative, the most desirable type of motion of the system is a damped oscillation or a simple decay without oscillation such as is sometimes produced when a jet is used to control the speed of rotation as in Michelson' $\mathrm{s}^{18}$ measurements of the velocity of light by means of a revolving mirror. In the so-called exact regulation the indicator returns to the normal setting after a single swing past it. It is generally, but not always, advantageous to eliminate all the variables but one which may be denoted by $x$, then, if $D \equiv d / d t$

$$
\left(p_{0} D^{n}+p_{1} D^{n-1}+\cdots+p_{n}\right) x=f(t) .
$$

The transient function $f(t)$ may be different from zero only for $0<t<T$. Then, for $t>T, x$ is a sum of terms satisfying the equation with $f(t)$ replaced by zero but it is not certain that all possible solutions of the homogeneous equation enter into the expression for the particular quantity $x$.

${ }^{16} \mathrm{~J}$. V. Poncelet, Cours de mêchanique, appliquée aux machines, Cours de l'école d'application de Metz, 1826. R. v. Mises, Dynamische Probleme der Maschinenlehre, Encyklopädie Mathematischen Wissenshaften vol. 4, part 10, pp. 153-355.

${ }^{17}$ M. Tolle, Die Regelung von Kraftmaschinen, 3d ed., Berlin, 1921. Tolle gives in particular a theory for the combination of a centrifugal governor and a relay. The theory is presented and amplified for the case of two relays and the effect of the steam by G. W. Higgs-Walker, Some problems connected with steam turbine governing, Proceedings of the Institute of Mechanical Engineers vol. 146 (1941) pp. 117-125.

${ }^{18} \mathrm{~A}$. A. Michelson, Measurements of the velocity of light between Mount Wilson and Mount San Antonio, Astrophysical Journal vol. 65 (1927) pp. 1-14; A. A. Michelson, F. G. Pease and F. Pearson, ibid. vol. 82 (1935) pp. 26-61. 
A regulator is said to be stable when, after a transient disturbance, the indicator returns to the region of insensitivity and does not go beyond this. In the case when the free motion involves an undamped or growing motion, the regulator is said to hunt or to be unstable. A sufficient condition for stability is that the roots of the algebraic equation

$$
F(z) \equiv p_{0} z^{n}+p_{1} z^{n-1}+\cdots+p_{n}=0
$$

should have only negative real parts. This condition may not be quite necessary because it may happen that a root with positive or zero real part does not happen to give a term in the expression for $x$. This possibility must be considered because something of an analogous nature seems to occur in some cases when the existence of a double root might make the sufficiency of the foregoing criterion seem doubtful.

The mathematical theory of stability based on the theory of small oscillations may be hard to use on account of lack of knowledge of the constants of the dynamical or electrical system. These can be estimated in many cases as in the theory of airplane stability but it is wise to have means of checking the results or of obtaining results when the computations are too difficult.

An oscillograph for the analysis of governor performance was built by J. E. Allen, ${ }^{19}$ and the East Pittsburgh Research Laboratories have built an instrument for analyzing governor performance which satisfies the specifications that have been laid down. Instruments of this nature have been made elsewhere. ${ }^{20}$ Dougill has devised an instrument for testing regulators in operation and has used it to test the governors in the gas works. ${ }^{21}$

2.2. Pseudo-negative roots. Liénard ${ }^{22}$ calls a quantity pseudo-negative when its real part is negative. The criterion for pseudo-negative roots of an algebraic equation is a special case of the criterion that the roots of an algebraic equation should lie within a specified circle in the complex plane, a line being regarded as a degenerate circle. A line may also be transformed into a circle by means of a transformation

${ }^{19} \mathrm{~J}$. E. Allen, Oscillograph analyses governor performance, Power vol. 78 (1934) pp. 610-612.

${ }^{20} \mathrm{~W}$. O. Oebon, A turbine governor performance analyzer, American Institute of Electrical Engineers vol. 69 (1941) pp. 963-967.

${ }^{21} \mathrm{G}$. Dougill, Retort house and exhauster governing of gas works, Engineering vol. 144 (1937) p. 697.

22 A. Liênard, Signe de la partie reélle des racines d'une équation algébrique, J. Math. Pures Appl. (9) vol. 15 (1936) pp. 235-250. 


$$
Z=(A z+B) /(C z+D)
$$

which does not change the degree of the equation

Cauchy ${ }^{23}$ devised a method for finding the criterion and Hermite ${ }^{24}$ carried the analysis much further considering particularly the conditions that the roots should lie in the upper half of the complex plane. He used a symmetrical polynomial

$$
H\left(z^{\prime}, z\right)=i\left[F\left(z^{\prime}\right) F_{0}(z)-F(z) F_{0}\left(z^{\prime}\right)\right] /\left(z-z^{\prime}\right)
$$

associated with the function $F(z)$ and an associated function $F_{0}(z)$ derived from $F(z)$ by changing $i$ into $-i$ in all the coefficients. The decomposition into squares of an associated quadratic form then indicated the number of pseudo-negative roots, this number being dependent in fact on the signature of the quadratic form.

At a meeting of the London Mathematical Society ${ }^{25}$ in 1868, James Clerk Maxwell asked if any member present could point out a way of determining in what cases all the possible parts of the imaginary roots of an algebraic equation are negative. He said that in studying the motion of certain governors for regulating machinery he had found that the stability of the motion depended on this condition, which is easily obtained for a cubic, but becomes difficult in the higher degrees. W. K. Clifford said in reply that by forming an equation whose roots are the sums of the roots of the original equation taken in pairs and by determining the condition of the real roots of this equation being negative, we should obtain the condition required.

Routh $^{26}$ used Clifford's idea when formulating conditions for a quartic equation with real coefficients,

$$
F(z)=a z^{4}+b z^{3}+c z^{2}+d z+e=0 .
$$

He says: "Let us form that symmetrical function of the roots which is the product of the sums of the roots taken two and two. If this be called $X / a^{3}$, we find $X=b c d-a d^{2}-e b^{2}$. Suppose we know the roots to be imaginary, say $\alpha \pm i p, \beta \pm i q$. Then

$$
X / a^{3}=4 \alpha \beta\left[(\alpha+\beta)^{2}+(p+q)^{2}\right]\left[(\alpha+\beta)^{2}+(p-q)^{2}\right] .
$$

${ }^{23}$ A. Cauchy, Calcul des indices des fonctions, J. Ecole Polytech. vol. 15 (1837) pp. 176-229, Oeuvres (2), vol. 1, pp. 416-466.

${ }^{24} \mathrm{C}$. Hermite, Extrait d'une lettre, Sur le nombre des racines d'une équation algébrique compris des limites données, J. Reine Angew. Math. vol. 52 (1856) pp. 39-51.

${ }^{25}$ See the discussion of the paper by J. J. Walker, On the anharmonic sextic, Proc. London Math. Soc. (1) vol. 2 (1868) pp. 60-61.

${ }^{26}$ E. J. Routh, Rigid dynamics, vol. 2, 1897, pp. 192-193; A treatise on the stability of motion, London, 1877; Advanced rigid dynamics, 6th ed., 1907, pp. 256-307. 
Thus, $\alpha \beta$ always takes the sign of $X / a$ and $\alpha+\beta$ always takes the sign of $-b / a$. The signs of both $\alpha$ and $\beta$ can therefore be determined; and if $a, b, X$ have the same sign, the real parts of the roots are all negative." Routh also formed the equation $G(z)=0$ whose roots are the sums in pairs of the roots of $F(z)=0$ and in the case when the coefficients $p_{r}$ in the general equation (2.1) are all real he came to the following conclusion:

In order that (2.1) may have all its roots pseudo-negative, it is necessary and sufficient that the equations $F(z)=0, G(z)=0$ should be complete with coefficients all of one sign. This means that no $p$ should be zero and that if $p_{0}>0$ then $p_{r}>0$. If, moreover, the coefficients of $G(z)$ are $q_{r}, r=0,1, \cdots, n^{2} / 2-n / 2$ then if $q_{0}>0$, we should also have $q_{r}>0$. These conditions give $n^{2} / 2+n / 2$ inequalities while the expected number of conditions is only $n$ so the foregoing conditions are not all independent.

In his work on governors in which he considered particularly the governors designed by Foucault and Lord Kelvin, Maxwell found that the stability depended upon the conditions for pseudo-negative roots of an equation of the fifth degree $z^{5}+p z^{4}+q z^{3}+r z^{2}+s z+t=0$. He found the necessary conditions $p q>r, p s<t$ but could not prove that these conditions were sufficient.

The simplicity of these conditions suggested that there might be necessary and sufficient conditions in the general case which could be formulated in a simple form. The subject of the stability of motion was soon afterwards proposed as a subject for the Adams Prize at the University of Cambridge and the prize was won by E. J. Routh of Peterhouse. He made use of the methods of Cauchy and Charles Sturm and a set of test functions was formed by a cascade process. Writing

$$
F(z)=E(z)+O(z)=A\left(z^{2}\right)+z B\left(z^{2}\right)
$$

so as to resolve $F(z)$ into its even and odd parts, he made use of the functions $s_{0}(y), s_{1}(y), s_{2}(y), \cdots, s_{n}(y)$, where

$$
s_{0}(y)=p_{0} y^{n}-p_{2} y^{n-1}+\cdots, s_{1}(y)=p_{1} y^{n-1}-p_{3} y^{n-3}+\cdots,
$$

$s_{2}(y)$ is the remainder with sign changed when use is made of the process for finding the G.C.M. of $s_{0}(y)$ and $s_{1}(y), s_{3}(y)$ is derived from $s_{1}(y)$ and $s_{2}(y)$ in a similar way, and so on. It is then clear as in Sturm's theorem that when $s_{r}(y)=0, s_{r+1}(y)$ and $s_{r-1}(y)$ have opposite signs. If $E$ denotes the excess of the number of changes of sign from + to in $s_{0}(y) / s_{1}(y)$ over that from - to + , then by Cauchy's theorem the whole number of radical points on the positive side of the axis of $y$ is 
$(n+E) / 2$. If $E=-n$ the roots are all pseudo-negative. The roots of the equations $A(x)=0, B(x)=0$ are in this case all negative and occur alternately. In the case of the biquadratic

$$
F(z)=z^{4}+p z^{3}+q z^{2}+r z+s=\left(z^{2}+x_{1}\right)\left(z^{2}+x_{2}\right)+p z\left(z^{2}+x_{0}\right)
$$

$q=x_{1}+x_{2}, r=p x_{0}, s=x_{1} x_{2}$ and Routh's test functions are

$s, p, p q-r=p\left(x_{1}+x_{2}-x_{0}\right), r(p q-r)-p^{2} s=p^{2}\left(x_{2}-x_{0}\right)\left(x_{0}-x_{1}\right)$.

The third test function is positive when and only when $x_{0}$ lies between $x_{1}$ and $x_{2}$. The test functions $p$ and $p q-r$ are both positive when $p>0$ and $x_{1}+x_{2}-x_{0}$ is positive. Now if $x_{1}-x_{0}$ is negative, $x_{2}$ must be positive. When $s$ is positive, $x_{1}$ and $x_{2}$ must be either both positive or both negative, hence if $x_{2}$ is positive so also is $x_{1}$. Routh's criteria for pseudo-negative roots imply then that $x_{0}, x_{1}, x_{2}$ are all positive and that $x_{0}$ lies between $x_{1}$ and $x_{2}$. When these conditions are all satisfied $s$ is positive, $r(p q-r)-p^{2} s$ is positive, $r(p q-r)$ and $q-r / p$ are positive. The ratio $r / p$ of the two last quantities is positive and so $q$ must be positive. If $p$ is also positive $r$ is positive. Routh's conditions are all satisfied and the equation $F(z)=0$ has pseudo-negative roots. Liénard attributes this converse theorem to $\mathrm{E}$. Jouguet and says that Chipart has extended it to an equation of any degree. A general proof is given in Liénard's paper.

Related polynomials such as $A(-x), B(-x)$ occur naturally in Rayleigh's theory of the driving point reaction in dynamics and in the theory of electric filters.

About 1893 the Swiss engineer Aurel Stodola ${ }^{27}$ investigated the stability of regulating devices for turbines, particularly those used in hydroelectric plants. He referred to Thomson and Tait's Natural philosophy ${ }^{28}$ for the relation between stability and pseudo-negative roots and on this account Corral ${ }^{29}$ has called the question of pseudonegative roots the problem of Lord Kelvin. Previously ${ }^{30}$ he had followed Orlando ${ }^{31}$ in calling it the problem of Hurwitz because

${ }_{27}$ A. Stodola, Über die Regulierung von Turbinen, Schweizerische Bauzeitung vol. 22 (1893) pp. 113-117, 121-122, 126-128, 134-135; vol. 23 (1894) pp. 108-112, 115-117.

${ }^{28}$ W. Thomson (Lord Kelvin) and P. G. Tait, Natural philosophy, vol. 1, 1879, p. 39.

${ }^{29} \mathrm{~J}$. J. Corral, Nueva solucion del problema de Lord Kelvin sobre ecuaciones de coefficientes reales, Revista de la Real Academia de Ciencias Exactas, Fisicas y Naturales de Madrid vol. 22 (1928) pp. 25-31.

${ }^{30} \mathrm{~J}$. J. Corral, Nuevos teoremas que resuelven el problema de Hurwitz, Madrid, Imprenta Clasica Española, 1921.

${ }^{31}$ L. Orlando, Sul problema di Hurwitz, Rendiconti Accademia Lincei (5) vol. 19 (1910) pp. 801-805; Math. Ann. vol. 71 (1911) pp. 233-245. 
Stodola's compatriot Adolf Hurwitz ${ }^{32}$ had investigated the subject and had succeeded in obtaining criteria in the determinantal form

$$
\begin{aligned}
p_{0}>0, & p_{1}>0, \quad\left|\begin{array}{ll}
p_{1} & p_{0} \\
p_{3} & p_{2}
\end{array}\right|>0, \quad\left|\begin{array}{lll}
p_{1} & p_{0} & 0 \\
p_{3} & p_{2} & p_{1} \\
p_{5} & p_{4} & p_{3}
\end{array}\right|>0, \\
& \left|\begin{array}{llll}
p_{1} & p_{0} & 0 & 0 \\
p_{3} & p_{2} & p_{1} & p_{0} \\
p_{5} & p_{4} & p_{3} & p_{2} \\
p_{7} & p_{6} & p_{5} & p_{4}
\end{array}\right|>0, \text { and so on. }
\end{aligned}
$$

The equivalence of the conditions of Routh and Hurwitz was shown by Bompiani, ${ }^{33}$ and Orlando obtained a proof by induction of the necessity and sufficiency of Hurwitz's conditions.

The study of equations with complex coefficients is also useful as there are some cases in which the conditions of Routh and Hurwitz are not the simplest possible conditions for pseudo-negative roots. In Appell's Mécanique rationnelle a discussion is given of dynamical equations such as

$$
\begin{aligned}
& x^{\prime \prime}+p_{1} x^{\prime}+q_{1} x-p_{2} y^{\prime}-q_{2} y=0, \\
& y^{\prime \prime}+p_{1} y^{\prime}+q_{1} y+p_{2} x^{\prime}-q_{2} x=0,
\end{aligned}
$$

in which there are gyrostatic terms. These equations are essentially those considered by Sir Horace Lamb in his work on kinetic stability ${ }^{34}$ and by $\mathrm{E}$. Jouguet in his work on secular stability. ${ }^{35}$ The algebraic equation obtained in the usual way is

$$
\left(z^{2}+p_{1} z+q_{1}\right)^{2}+\left(p_{2} z+q_{2}\right)^{2}=0
$$

but if we put $x+i y=Z$, as Lamb does, there is a single dynamical equation

$$
Z^{\prime \prime}+\left(p_{1}+i p_{2}\right) Z^{\prime}+q_{1}+i q_{2}=0
$$

\footnotetext{
${ }^{22}$ A. Hurwitz, Über die Bedingungen, unter welchen eine Gleichung nur Wurzeln mit negativen reellen Theilen besitzt, Math. Ann. vol. 46 (1895) pp. 273-284; Werke, vol. 2, pp. 533-545.

${ }^{33} \mathrm{E}$. Bompiani, Sulle condizioni sotto le quali un equazione a coefficienti reale ammette solo radici con parte reale negative, Giornale di Matematica vol. 49 (1911) pp.3339.

« H. Lamb, On kinetic stability, Proc. Roy. Soc. London. Ser. A. vol. 80 (1908) pp. $168-177$.

${ }^{35}$ E. Jouquet, Sur la stabilité seculaire quand les forces positionnelles n'admettent pas de potentiel, C. R. Acad. Sci. Paris vol. 207 (1938) pp. 267-270.
} 
which gives rise to an algebraic equation

$$
z^{2}+\left(p_{1}+i p_{2}\right) z+q_{1}+i q_{2}=\left(z+x_{1}+i y_{1}\right)\left(z+x_{2}+i y_{2}\right)=0 \text {. }
$$

The conditions for pseudo-negative roots are now

$$
x_{1}+x_{2}>0 \text { and } x_{1} x_{2}\left[\left(x_{1}+x_{2}\right)^{2}+\left(y_{1}-y_{2}\right)^{2}\right]>0
$$

while the corresponding conditions derived by considering the biquadratic equation are $x_{1}+x_{2}>0$ and

$$
x_{1} x_{2}\left[\left(x_{1}+x_{2}\right)^{2}+\left(y_{1}-y_{2}\right)^{2}\right]\left[\left(x_{1}+x_{2}\right)^{2}+\left(y_{1}+y_{2}\right)^{2}\right]>0
$$

and there is an extra factor in the expression used for the second criterion.

The conditions for the quadratic may be expressed in terms of the quantities $I_{1}, I_{2}, I_{3}$ which are invariant when the equation is changed into a new equation by a substitution of the form $z=Z+i a$, where $a$ is real. If

$$
\begin{aligned}
(Z+i a)^{2}+\left(p_{1}+i p_{2}\right)(Z+i a)+q_{1} & +i q_{2} \\
& \equiv Z^{2}+\left(P_{1}+i P_{2}\right) Z+Q_{1}+i Q_{2}
\end{aligned}
$$

then $P_{1}=p_{1}, P_{2}=p_{2}-2 a, Q_{1}=q_{1}+a p_{2}-a^{2}, Q_{2}=q_{2}-a p_{1}$ and so there are 3 invariants

$$
\begin{gathered}
I_{1}=P_{1}=p_{1}, \quad I_{2}=Q_{1}+P_{2}^{2} / 4=q_{1}+p_{2}^{2} / 4, \\
I_{3}=Q_{2}-P_{1} P_{2} / 2=q_{2}-p_{1} p_{2} / 2 .
\end{gathered}
$$

If, in particular, we choose $a$ so that $P_{2}=0$, the equation takes the simple form

$$
Z^{2}+I_{1} Z+I_{2}+i I_{3}=0
$$

If $Z_{1}, Z_{2}$ are the roots of this equation and if $T_{1}, T_{2}$ are the roots of the conjugate equation

$$
T^{2}+I_{1} T+I_{2}-i I_{3}=0,
$$

the equation whose roots are $Z_{1}+T_{1}, Z_{2}+T_{2}, Z_{1}+T_{2}, Z_{2}+T_{1}$ is

$$
S^{4}+4 I_{1} S^{8}+\left(5 I_{1}^{2}+4 I_{2}\right) S^{2}+\left(2 I_{1}^{3}+8 I_{1} I_{2}\right) S+4 I_{1}^{2} I_{2}-4 I_{3}^{2}=0 \text {. }
$$

This is also the equation whose roots are $z_{1}+t_{1}, z_{2}+t_{2}, z_{1}+t_{2}, z_{2}+t_{1}$ where $z_{1}, z_{2}$ are the roots of the original equation and $t_{1}, t_{2}$ are the roots of its conjugate equation. It should be noticed that the terms in the equation for $S$ involve the invariants and $S$ only, moreover, by using two of these terms expressions 


$$
\left.x_{1}+x_{2}=I_{1}, \quad x_{1} x_{2} \mathrm{~L}\left(x_{1}+x_{2}\right)^{2}+\left(y_{1}-y_{2}\right)^{2}\right]=4\left(I_{1}^{2} I_{2}-I_{3}^{2}\right)
$$

are obtained for the quantities that furnish criteria for the roots to be pseudo-negative.

The quadratic equation may be reduced to a canonical form

$$
\frac{1}{z+i w_{1}}+\frac{1}{z+i w_{2}}+\frac{1}{z+u+i v}=0
$$

where $w_{1}, w_{2}, u$ and $v$ are real quantities. The equation is then of stable type (with pseudo-negative roots) when $u>0$ for the roots are those of the derived function of the cubic

$$
(z+i w)(z+i w)(z+u+i v)=0
$$

and so by the theorem of Gauss ${ }^{36}$ and Lucas $^{37}$ lie within the triangle formed by the points $z=-i w_{1}, z=-i w_{2}, z=-u-i v$ in the complex $z$-plane. The roots are in fact the foci of the ellipse which touches the sides of this triangle at its middle points.

The equations for determining $u, v, w_{1}, w_{2}$ are

$$
\begin{gathered}
3 p_{1}=2 u, \quad 3 p_{2}=2\left(v+w_{1}+w_{2}\right), \quad 3 q_{1}=-w_{1} w_{2}-v\left(w_{1}+w_{2}\right), \\
3 q_{2}=u\left(w_{1}+w_{2}\right)
\end{gathered}
$$

and so $w_{1}+w_{2}=2 q_{2} / p_{1}, w_{1} w_{2}=-3 q_{1}-\left(3 p_{1} p_{2} q_{2}-4 q_{2}^{2}\right) / p_{1}^{2}$. The quantities $w_{1}, w_{2}$ are thus the roots of the quadratic equation

$$
p_{1}^{2} w^{2}-2 q_{2} p_{1} w+4 q_{2}^{2}-3 p_{1} p_{2} q_{2}-3 p_{1}^{2} q_{1}=0
$$

which has real roots when $p_{1}^{2} q_{1}+p_{1} p_{2} q_{2}-q_{2}^{2}>0$ or $I_{1}^{2} I_{2}-I_{3}^{2}>0$. When this condition is satisfied the sign of $u$ is positive when $I_{1}>0$.

The extension of Clifford's method which was used for the quadratic may be applied also to the cubic

$$
z^{3}+\left(p_{1}+i p_{2}\right) z^{2}+\left(q_{1}+i q_{2}\right) z+r_{1}+i r_{2}=0 .
$$

The 5 invariants are $I_{1}=p_{1}, 3 I_{2}=p_{2}^{2}+3 q_{1}, 3 I_{3}=3 q_{2}-2 p_{1} p_{2}, 3 I_{4}=9 r_{1}$ $-3 p_{1} q_{1}+3 p_{2} q_{2}-2 p_{1}^{2} p_{2}^{2}, 27 I_{5}=27 r_{2}-9 p_{2} q_{1}-2 p_{2}^{3}$. When a substitution $z=Z+i a$ is chosen so that in the new equation the coefficient of $Z^{2}$ is real, the new equation is

$$
Z^{3}+I_{1} Z^{2}+\left(I_{2}+i I_{3}\right) Z+\left(I_{4}+I_{1} I_{2}\right) / 3+i I_{5}=0 .
$$

36 C. F. Gauss, Oeurves, vol. 3, 1886, p. 112; vol. 8, 1900, p. 32.

${ }^{37}$ F. Lucas, Géometrie des polynômes, J. École Polytech. vol. 29 (1879) pp. 1-33. See also M. Marden, The location of the zeros of the derivative of a polynomial, Amer. Math. Monthly vol. 42 (1935) pp. 277-286. 
If the roots of this equation are $Z_{1}, Z_{2}, Z_{3}$ and those of the conjugate equation $T_{1}, T_{2}, T_{3}$ the equation for $S=Z+T$ may be readily found by elimination and its roots are the 9 quantities $Z_{1}+T_{1}, Z_{1}+T_{2}$, $Z_{1}+T_{3}, Z_{2}+T_{1}, Z_{2}+T_{2}, Z_{2}+T_{3}, Z_{3}+T_{1}, Z_{3}+T_{2}, Z_{3}+T_{3}$. The product of the roots of this equation is particularly interesting as it furnishes a quantity

$$
\begin{aligned}
K & =J\left(I_{1} I_{2}-J\right)^{2}-3 J I_{1} I_{3} I_{5}+I_{1}^{2} I_{2} I_{3} I_{5}-J I_{2} I_{3}^{2}-I_{1}^{3} I_{5}^{2}, \\
J & =I_{4}+I_{1} I_{2},
\end{aligned}
$$

which is positive when all the roots are pseudo-negative. The necessary and sufficient conditions for pseudo-negative roots are $I_{1}>0$, $K>0$, and $I>0$ where these quantities are such that when positive they imply that $X_{1}+X_{2}+X_{3}, X_{1} X_{2} X_{3}$ and $X_{2} X_{3}+X_{3} X_{1}+X_{1} X_{2}$ are all positive. To find the invariant $I$ it is helpful to use the notation

$$
\begin{aligned}
U_{1}= & X_{1}+i Y_{1}, \quad U_{2}=X_{2}+i Y_{2}, \quad U_{3}=X_{3}+i Y_{3}, \quad V_{1}=X_{1}-i Y_{1}, \\
V_{2}= & X_{2}-i Y_{2}, \quad V_{3}=X_{3}-i Y_{3}, \quad H_{r s}=U_{r}+V_{8} . \\
I= & \left(H_{22} H_{33}+H_{33} H_{11}+H_{11} H_{22}\right)\left(H_{23} H_{31}+H_{31} H_{12}\right. \\
& \left.+H_{12} H_{23}\right)\left(H_{32} H_{13}+H_{13} H_{21}+H_{21} H_{32}\right) \\
= & 4\left(X_{2} X_{3}+X_{3} X_{1}+X_{1} X_{2}\right)\left|H_{23} H_{31}+H_{31} H_{12}+H_{12} H_{23}\right|^{2} .
\end{aligned}
$$

The quantities $U_{1}, U_{2}, U_{3}$ are identical with $-Z_{1},-Z_{2}$ and $-Z_{3}$; also

$$
I=\left(W-W_{1}\right)\left(W-W_{2}\right)\left(W-W_{3}\right)
$$

where

$$
\begin{aligned}
W_{1}= & U_{2} U_{3}+U_{3} U_{1}+U_{1} U_{2}+V_{2} V_{3}+V_{3} V_{1}+V_{1} V_{2} \\
& +\left(U_{1}+U_{2}+U_{3}\right)\left(V_{1}+V_{2}+V_{3}\right), \\
W_{1}= & U_{1} V_{1}+U_{2} V_{2}+U_{3} V_{3}, \quad W_{2}=U_{2} V_{3}+U_{3} V_{1}+U_{1} V_{2}, \\
W_{3}= & U_{3} V_{2}+U_{1} V_{3}+U_{2} V_{1} .
\end{aligned}
$$

These quantities $W_{1}, W_{2}, W_{3}$ and a second set of quantities $W_{1}^{\prime}, W_{2}^{\prime}$, $W_{3}^{\prime}$ obtained by changing the cyclic order of $V_{1}, V_{2}, V_{3}$ to $V_{3}, V_{2}, V_{1}$ are the roots of a sextic equation

$$
\left(W^{3}-A W^{2}+B W-C\right)^{2}=d^{2} D D^{\prime}
$$

where $D$ is the discriminant of the equation for $U$ and $D^{\prime}$ is the discriminant of the equation for $V$. This form is indicated by the fact that when the equation for $U_{1}, U_{2}, U_{3}$ has equal roots or when the equation for $V_{1}, V_{2}, V_{3}$ has equal roots the two sets of three sums become the same. Also we have identically $W_{1}^{\prime}+W_{2}^{\prime}+W_{3}^{\prime}=W_{1}+W_{2}$ 
$+W_{3}, W_{2}^{\prime} W_{3}^{\prime}+W_{3}^{\prime} W_{1}^{\prime}+W_{1}^{\prime} W_{2}^{\prime}=W_{2} W_{3}+W_{3} W_{1}+W_{1} W_{2}$.

To find $d$ we put $W=0$ and note that $W_{1} W_{2} W_{3}-W_{1}^{\prime} W_{2}^{\prime} W_{3}^{\prime}$ $=2 d\left(D D^{\prime}\right)^{1 / 2}$. But

$W_{1} W_{2} W_{3}-W_{1}^{\prime} W_{2}^{\prime} W_{3}^{\prime}$

$$
=\left(U_{2}-U_{3}\right)\left(U_{3}-U_{1}\right)\left(U_{1}-U_{2}\right)\left(V_{2}-V_{3}\right)\left(V_{3}-V_{1}\right)\left(V_{1}-V_{2}\right)=\left(D D^{\prime}\right)^{1 / 2} \text {, }
$$

consequently $2 d=1$. We also have the relation

$$
\begin{aligned}
2 C= & W_{1} W_{2} W_{3}+W_{1}^{\prime} W_{2}^{\prime} W_{3}^{\prime} \\
= & U_{1} U_{2} U_{3}\left(V_{1}^{3}+V_{2}^{3}+V_{3}^{3}\right)+V_{1} V_{2} V_{3}\left(U_{1}^{3}+U_{2}^{3}+U_{3}^{3}\right)+6 U_{1} U_{2} U_{3} V_{1} V_{2} V_{3} \\
& +\left(U_{2}^{2} U_{3}+U_{3}^{2} U_{2}+U_{3}^{2} U_{1}+U_{1}^{2} U_{3}+U_{1}^{2} U_{2}+U_{2}^{2} U_{1}\right)\left(V_{2}^{2} V_{3}+V_{3}^{2} V_{2}\right. \\
& \left.+V_{3}^{2} V_{1}+V_{1}^{2} V_{3}+V_{1}^{2} V_{2}+V_{2}^{2} V_{1}\right) \\
= & R\left(P^{\prime 3}-3 P^{\prime} Q^{\prime}+3 R^{\prime}\right)+R^{\prime}\left(P^{3}-3 P Q+3 R\right)+6 R R^{\prime} \\
& +(P Q-3 R)\left(P^{\prime} Q^{\prime}-3 R^{\prime}\right)
\end{aligned}
$$

where $P, Q, R$ are the coefficients in the equation for $Z_{1}, Z_{2}, Z_{3}$, $P^{\prime}, Q^{\prime}, R^{\prime}$ the coefficients in the equation for $T_{1}, T_{2}, T_{3}$.

Hence $2 \mathrm{C}=R P^{\prime 3}+R^{\prime} P^{3}-6 P^{\prime} Q^{\prime} R-6 P Q R^{\prime}+P Q P^{\prime} Q^{\prime}+18 R R^{\prime}$. Also

$$
\begin{aligned}
B= & W_{2} W_{3}+W_{3} W_{1}+W_{1} W_{2}=\left(U_{1}^{2}+U_{2}^{2}+U_{3}^{2}\right)\left(V_{2} V_{3}+V_{3} V_{1}+V_{1} V_{2}\right) \\
& +\left(U_{2} U_{3}+U_{3} U_{1}+U_{1} U_{2}\right)\left(V_{2} V_{3}+V_{3} V_{1}+V_{1} V_{2}+V_{1}^{2}+V_{2}^{2}+V_{3}^{2}\right) \\
= & Q^{\prime}\left(P^{2}-2 Q\right)+Q\left(P^{\prime 2}-Q^{\prime}\right)=P^{2} Q^{\prime}+P^{\prime 2} Q-3 Q Q^{\prime} .
\end{aligned}
$$

Hence

$$
\begin{aligned}
& \left(W-W_{1}\right)\left(W-W_{2}\right)\left(W-W_{3}\right)=W^{3}-P P^{\prime} W^{2}+\left(P^{2} Q^{\prime}+P^{\prime 2} Q-3 Q Q^{\prime}\right) W \\
& \quad-\left(R P^{\prime 3}+R^{\prime} P^{3}-6 P^{\prime} Q^{\prime} R-6 P Q R^{\prime}+P Q P^{\prime} Q^{\prime}+18 R R^{\prime}\right) / 2-\left(D D^{\prime}\right)^{1 / 2} / 2 .
\end{aligned}
$$

With the value $W=Q+Q^{\prime}+P P^{\prime}$ the expression for $I$ is

$$
\begin{aligned}
I= & \left(Q+Q^{\prime}+P P^{\prime}\right)^{3}-P P^{\prime}\left(Q+Q^{\prime}+P P^{\prime}\right)^{2} \\
& +\left(P^{2} Q^{\prime}+P^{\prime 2} Q-3 Q Q^{\prime}\right)\left(Q+Q^{\prime}+P P^{\prime}\right) \\
& -\left(R P^{\prime 3}+R^{\prime} P^{3}-6 P^{\prime} Q^{\prime} R-6 P Q R^{\prime}+P Q P^{\prime} Q^{\prime}+18 R R^{\prime}\right) / 2-\left(D D^{\prime}\right)^{1 / 2} / 2 .
\end{aligned}
$$

This is the expression obtained in a former paper. ${ }^{38}$ It is well known that

$$
D=P^{2} Q^{2}-4 P^{3} R+18 P Q R-27 R^{2}
$$

while $D^{\prime}$ can be expressed in a similar way in terms of $P^{\prime}, Q^{\prime}, R^{\prime}$. It should be mentioned that the conditions for pseudo-negative roots for

${ }^{38} \mathrm{H}$. Bateman, Stability of the parachute and helicopter, National Advisory Committee for Aeronautics, Report No. 80, 1920. 
the case of the cubic equation with complex coefficients should be derivable from the conditions given by $\mathrm{P}$. Bohl ${ }^{39}$ that the roots of a trinomial equation may have moduli less than $p$.

2.3. The case of equal roots. In the solution of linear differential equations with constant coefficients a double root of the associated algebraic equation indicates the existence of secular terms such as $a \sin (m t)+b t \cos (m t)$ or $(A+B t) e^{-k t}$ in the general solution. This fact was a kind of bogie in the theory of the small oscillations of a dynamical system because it was thought at one time that there was a kind of instability associated with the presence of repeated roots. In 1858 Weierstrass ${ }^{40}$ completed the theory of normal coordinates and showed that in some cases at least secular terms do not occur in the final solution of the equations of motion. Further remarks of interest were made by Somoff, ${ }^{41}$ Routh ${ }^{42}$ and Stokes. ${ }^{43} \mathrm{~A}$ review of the subject has been given recently by Melikov. ${ }^{44}$

Experience shows that it is better to work with the original system of differential equations than with the single equation obtained by eliminating all the variables but one. For instance, in the case of the well known system

$$
a\left(x^{\prime \prime}+k^{2} x\right)=b\left(y^{\prime \prime}+k^{2} y\right)=c\left(z^{\prime \prime}+k^{2} z\right)=b c x+c a y+a b z=s,
$$

say, the algebraic equation for $m$ in an exponential factor $e^{m t}$ occurring in the solutions is $\left(m^{2}+k^{2}\right)^{2}\left(m^{2}+h^{2}\right)=0$ where $h^{2}=k^{2}-b c / a-c a / b-a b / c$. The equation for $s$ is, however, $s^{\prime \prime}+h^{2} s=0$ and so secular terms do not appear when $s$ is calculated first and $x, y, z$ derived from $s$.

\footnotetext{
${ }^{39}$ P. Bohl, Zur Theorie der trinomischen Gleichungen, Math. Ann. vol. 56 (1908) pp. 556-569.

${ }^{40} \mathrm{~K}$. Weierstrass, Über ein die homogenen Functionen zweiten Grades betreffendes Theorem, nebst Anwendung desselben auf die Theorie der kleinen Schwingungen, Monatsberichte der Akademie der Wissenschaft zu Berlin, 1858, pp. 207-220; Mathematische Werke, vol. 1, Berlin, 1894, pp. 233-246. See also F. Purser, Occurrences of equal roots in Lagrange's determinantal equation, Report of the British Association for the Advancement of Science, 1878, pp. 463-464.

${ }^{41} \mathrm{~K}$. Somoff, Oscillations of systems of particles, algebraic problem, Mémoires de l'Academie des Sciences de Saint Petersburg (Akademiia Nauk), no. 14, 1879, 30 pp. The related paper of C. Jordan, Sur les oscillations infiniment petites des systemes matériels, C. R. Acad. Sci. Paris vol. 74 (1872) pp. 1395-1399, is reviewed unfavorably in Fortschritte der Mathematik vol. 4 (1872) pp. 471-472.

12 E. J. Routh, Rigid dynamics, part 2, pp. 84, 190.

${ }^{43} \mathrm{G}$. G. Stokes, Explanation of a dynamical paradox, Messenger of Mathematics vol. 1 (1872) pp. 1-3; Mathematical and physical papers, vol. 4, pp. 334-335.

${ }^{44} \mathrm{~K}$. V. Melikov, Über das Theorem von Weierstrass und Routh, Annals of the Institute of Mines, Leningrad, vol. 10 (1936) pp. 71-76.
} 
The case of equal roots is connected of course with the phenomenon of resonance and there are many cases in practice in which a number of identical dynamical systems are coupled together particularly in the construction of acoustical, electrical and mechanical filters. Much depends on the nature of the coupling and even when equal roots do not occur in the final analysis there are interesting phenomena. The use of symmetrical arrangements is sometimes advantageous on account of the simplicity of the analysis.

In gas producing plants in which there is one exhaust regulator for two coke ovens there seems to be an idea that symmetry must be avoided on account of a possible interaction or resonance between two coke ovens which would make the regulator unstable. Thus Dougill ${ }^{45}$ remarks: "The interaction which so often occurred when two retort houses of equal size were connected to a common main which led to one exhaust governor could be remedied by provision of a time lag, preferably in the exhaust governor."

The question may be raised whether the troubles encountered can really be attributed to the equality in size and an answer to this question cannot be given without a careful analysis of the precise setup. In the meantime, however, it may be of interest to examine some of the complications which arise when use is made of a time lag in dynamical or electrical systems.

\section{The tRANSCENDENTAL PROBLEM}

3.1. Time lag in control systems. Long ago the delayed action of a regulating system was recognized as one of the primary causes of the hunting of governed engines. ${ }^{46}$ The effect of time lag has consequently been studied by many investigators, particularly by D. R. Hartree, A. Porter, A. Callender, A. B. Stevenson, ${ }^{47}$ H. König, ${ }^{48}$ J. G. Ziegler

${ }^{45}$ G. Dougill, Retort house and exhauster governing of gas works, Engineering vol. 144 (1937) p. 144.

${ }^{46}$ See for instance, J. Swinburne, The hunting of governed engines, Engineering vol. 58 (1894) p. 247; Practice, The "hunting" of steam engine governors, Engineering vol. 71 (1901) p. 216.

${ }^{47}$ A. Callender, D. R. Hartree and A. Porter, Time-lag in a control system, Trans. Roy. Soc. London Ser. A. vol. 235 (1936) pp. 415-444. D. R. Hartree, A. Porter, A. Callender and A. B. Stevenson, Time-lag in a control system. II, Proc. Roy. Soc. London Ser. A. vol. 161 (1937) pp. 460-476. A. Callender and A. B. Stevenson, Proceedings of the Society of the Chemical Industry (Chemical Engineering Group) vol. 18 (1936) p. 108. See also L. Nisolle, Sur la stabilitê des rêgulateurs d impulsions retardies ou amorties, C. R. Acad. Sci. Paris vol. 211 (1940) pp. 762-765.

${ }^{48} \mathrm{H}$. König, Periodische und aperiodische Schwingungen an empfindlichen Regelan ordnungen, Zeitschrift für Technische Physik vol. 18 (1937) pp. 426-431. See also D. Stein, Untersuchung der Stabilitätsbedingungen bei verzögerter Regelung, Elektrische Nachrichten Technik vol. 20 (1943) pp. 205-213. 
and N. B. Nichols. ${ }^{49}$ Time lag was also considered by H. L. Hazen ${ }^{50}$ in his work on servo mechanisms and by N. Minorsky ${ }^{51}$ in his study of control problems.

The effects of time lag have been considered usually by three distinct methods :

(1) By the use of Taylor's theorem and a neglect of small terms so that linear differential equations are obtained.

(2) By the use of differential difference equations or equations of mixed differences.

(3) By the use of integral equations of the Poisson-Volterra type.

The first method is explained in a general discussion of control problems by the editorial staff of The Engineer and by N. Minorsky who regards the differential equation as an asymptotic form and gives four different types. In a simple case a body is supposed to oscillate under the influence of a restoring force $R(t-k)$ proportional to the body's displacement at a previous instant and also under the influence of a damping depending partly on the body's instantaneous velocity and partly on its velocity at a previous time $t-h$. The equation of motion is supposed, indeed, to be of the type ${ }^{52}$

$$
x^{\prime \prime}+Q x^{\prime}+N f^{\prime}(t-h)+P f(t-k)=0
$$

where the time lags $h$ and $k$ are regarded as independent of $t$. In the approximate theory $f^{\prime}(t-h)$ and $f(t-k)$ are replaced by $f^{\prime}(t)-h f^{\prime \prime}(t)$ and $f(t)-k f^{\prime}(t)+k^{2} f^{\prime \prime}(t) / 2$ respectively and then $x$ is used in place of $f(t)$. The resulting equation is

$$
\left(1-N h+P k^{2} / 2\right) x^{\prime \prime}+(Q+N-P k) x^{\prime}+P x=0 .
$$

When $Q=N=0$ the lag in $R$ gives a negative damping and so oscillation with increasing amplitude may be expected to occur. When $N=0$ and $Q \not \equiv 0$ the negative damping may be overcome by positive damping depending on $Q$. When $N=0$ both $N$ and $Q$ may tend to overcome the negative damping but if $h$ is large the coefficient of $x^{\prime \prime}$ may become negative and completely alter the character of the motion. Minorsky ${ }^{53}$ indicates an asymptotic form in which the apparent re-

49 J. G. Ziegler and N. B. Nichols, Process lags in automatic-control circuits, Transactions of the American Society of Mechanical Engineers vol. 65 (1943) pp. 433-444.

${ }^{50}$ H. L. Hazen, Servo mechanisms, Journal of the Franklin Institute vol. 218 (1934) pp. 279-331, 543-580.

${ }^{51}$ N. Minorsky, Control problems, ibid. vol. 232 (1941) pp. 451-488.

${ }^{52}$ Editorial Staff, The damping effect of time lag, The Engineer vol. 163 (1937) p. 439.

${ }^{63} \mathrm{~N}$. Minorsky, Self-excited oscillations in dynamical systems possessing retarded actions, Transactions of the American Society of Mechanical Engineers vol. 64 (1942) pp. A65-A71, discussion by H. Poritzky, pp. A195-A196. 
storing term is different from $P x$, but by the multiplication of the equation by a suitable factor this form may be reduced to the previous ones in which only the coefficients of $x^{\prime}$ and $x^{\prime \prime}$ are affected by the lags.

The indications of the approximate theory need to be checked by exact analysis but they are such as to make it plain that the effects of time lag may be quite serious.

When there is a single time lag which is treated as constant some progress may be made with the aid of the known theory of linear equations of mixed differences. The particular equations discussed by Hartree and his collaborators are

$$
\begin{aligned}
u^{\prime}(x) & =f(x)+v(x)-c u(x), \\
-v^{\prime}(x+1) & =p u(x)+q u^{\prime}(x)+r u^{\prime \prime}(x)
\end{aligned}
$$

where $p, q, r$ and $c$ are real constants. When $f(x)=0$ the free motion is described by means of terms of type $u(x)=K$ exp $(k x)$ where $k$ is determined by means of the transcendental equation

$$
k(k+c)=e^{-k}\left(p+q k+r k^{2}\right) .
$$

This equation and some related equations are discussed chiefly by graphical methods but for the equation

$$
z^{2} e^{z}+a_{0} z+a_{1}=0
$$

an approximate solution $z=\log \left(a_{0} / b\right)+i b, b=(2 n+1 / 2) \pi+b^{-1}[\log$ $\left.\left(a_{0} / b\right)-a_{1} / a_{0}\right]$, is given for the value of $z$ for a high harmonic on the supposition that $b$ is large compared with $a_{1} / a_{0}$ and the real part of z. This approximation may hold in some cases for the fundamental and if it does it indicates that if the fundamental is positively damped the higher harmonics are more strongly damped.

Transcendental equations of the form $e^{z}=$ rational function of $z$ are of frequent occurrence. A simple equation of this type $z=a-c e^{-z}$ occurs in economics ${ }^{54}$ in the work of Kalecki, Frisch, Holme, James and Belz. It is a generalization of an equation considered by $\mathrm{Euler}^{55}$ in 1750.

In his discussion of control problems König avoids the assumption

${ }^{34}$ M. Kalecki, $A$ macrodynamic theory of business cycles, Econometrica vol. 3 (1935) pp. 327-344. R. Frisch and H. Holme, ibid. pp. 225-239. R. W. James and M. H. Belz, ibid. vol. 4 (1936) pp. 157-160.

${ }_{{ }_{5}} \mathrm{~L}$. Euler, Investigatio curvarum quae evolutae sui similes producunt, Akademiia Nauk vol. 12 (1750) pp. 3-52. See also M. Alle, Ein beitrag zur Theorie der Evoluten, Akademie der Wissenschaften Wien (IIa) vol. 113 (1904) pp. 53-70. 
of a constant time-lag and obtains an integral equation of Poisson's type,

$$
x(t)+m \int_{0}^{t} k(t-T) x(T) d T=x_{0}(t),
$$

where $x(t)$ is the variable quantity to be regulated, $x_{0}(t)$ is the variation of this quantity when there is no control, $m$ is the factor of amplification and $k(t)$ is a function of type $I^{\prime}(t)$, where $I(t)$ is the influence function which electrical engineers call the transfer function. The function $k(t)$ is generally zero up to time $t_{0}$, it then rises gradually in value until it reaches a peak value and then remains practically constant from a time $t_{1}$ on. The graph of $k(t)$ generally has a peak but in a simple case worked out by König, $k(t)$ is constant for $t_{0}<t<t_{1}$ and is zero for other values of $t$. In a stable kind of regulation the free motion with $x_{0}(t)=0$ is damped. When undamped oscillations or growing oscillations can arise the system may be capable of spontaneous oscillation.

König seeks the condition that there may be a solution of type $x(t)=A \exp (i w t-h t)$ and obtains the conditions

$$
\int k(u) e^{h u} \sin (w u) d u=0, \quad \int k(u) e^{h u} \cos (w u) d u=0 .
$$

The limit of stability is then given by $h=0$. The motion due to a transitory disturbance may be found by Poisson's method of successive approximation $s^{58}$ in which $x(t)$ is expanded in powers of $m$ or it may be found by a method recommended by V. Pareto ${ }^{57}$ and the present author ${ }^{58}$ in which a relation is found between the generating functions

$$
\begin{gathered}
X(z)=\int_{0}^{\infty} e^{-z t} x(t) d t, \quad K(z)=\int_{0}^{\infty} e^{-z t} k(t) d t, \\
X_{0}(z)=\int_{0}^{\infty} e^{-z t} x_{0}(t) d t .
\end{gathered}
$$

${ }^{B 6} \mathrm{~S}$. D. Poisson, Mémoire sur la thêorie du magnétisme en mouvement, Académie des Sciences, Paris, 1826, 130 pp. (pp. 28-30).

${ }^{87}$ V. Pareto, Sur les fonctions génératrices d'Abel, J. Reine Angew. Math. vol. 110 (1892) pp. 29-323.

${ }^{88} \mathrm{H}$. Bateman, Report on the history and present state of the theory of integral equations, British Association for the Advancement of Science, 1910, pp. 345-424 (p. 394); An integral equation occurring in a mathematical theory of retail trade, Messenger of Mathematics vol. 49 (1920) pp. 1-4. 
3.2. Feedback. An early use of feedback to regulate a water clock has been ascribed to James Watt ${ }^{59}$ who apparently used a pump to maintain the desired constant level in the reservoir from which the water flows. The rate of flow should be constant if the level of the water in a receiving vessel is to give a correct measure of time. A cascade system of reservoirs which kept this rate very nearly constant for a short time was adopted long ago in the design of a water clock of Canton, China, known as "Hon-woo-et-low" (copper jars dropping water). James Arthur ${ }^{60}$ saw this clock in 1897 and was told that it had been in existence for over 3000 years, being known as the clock of the street arch.

A mathematical theory of a cascade system of reservoirs based upon the formula for the discharge of a weir was given by E. Maillet ${ }^{61}$ about 1905 . The system of differential equations is nonlinear but some interesting conclusions are drawn relating to the existence of a steady state and the manner in which it is approached. The problem of stability of the steady state is considered and some attention is given also to the case in which water is fed into the reservoirs from an outside source. When in addition feedback is introduced there are many mathematical problems to be solved. Maillet's analysis is of some mathematical interest as it led him to researches on almost periodic functions.

Feedback has been much used in recent years in systems employing vacuum tubes and amplifiers. In his description of stabilized feedback amplifiers H. S. Black ${ }^{62}$ says: "By building an amplifier whose gain is deliberately made, say, 40 decibels higher than necessary (10000 fold excess on energy basis) and then feeding the output back on the input in such a way as to throw away the excess gain, it has been found possible to effect extraordinary improvement in constancy of amplification and freedom from nonlinearity."

In the simplified mathematical theory which has been developed by

${ }^{59}$ See the discussion by Field of the paper by J. Woods, Exhibition and description of the chronometric governor, invented by Messrs $E$. W. and $C$. W. Siemens, Minutes and Proceedings of the Institute of Civil Engineers, London, vol. 5 (1846) pp. 255-265.

60 James Arthur, Time and its measurement, Windsor, Chicago, 1909.

61 E. Maillet, Sur la vidage des systèmes de réservoirs, C. R. Acad. Sci. Paris vol. 140 (1905) pp. 712-714; Sur les équations différentielles et les systèmes de réservoirs, ibid. vol. 147 (1908) pp. 966-968; Sur les systèmes de réservoirs, ibid. vol. 149 (1909) pp. 105-107. See also Bull. Soc. Math. France vol. 33 (1905) pp. 129-145; Annales des Ponts et Chaussées (1906) pp. 110-149; J. École Polytech. (2) vol. 13 (1909) pp. 27-56; J. Math. Pures Appl. (6) vol. 9 (1913) pp. 171-231.

${ }^{62}$ H. S. Black, Stabilized feedback amplifiers, Bell System Technical Journal vol. 13 (1934) pp. 1-18. 
the electrical engineers ${ }^{63}$ the effects of inertia or induction are neglected and the electrical system is supposed to be built up from simple delay elements and elements with a constant type of amplification over a limited range. The result of feedback is thus represented by an equation

$$
x=-Q x
$$

where $x$ denotes the amplification factor which is the product $m_{1} m_{2} \cdots m_{n}$ of a number of individual amplification factors and $Q$ is the product of a number of differential operators of type

$$
q=1 /(1+\tau p), \quad p=d / d t .
$$

The differential equation for $x$ is

$$
\left[\left(1+\tau_{1} p\right)\left(1+\tau_{2} p\right) \cdots\left(1+\tau_{n} p\right)+\mu\right] x=0
$$

and when a particular solution is of form $x=a e^{z t}, z$ satisfies the algebraic equation obtained by replacing $p$ by $z$. A graphical method of finding the condition for stability has been given by H. Nyquist. ${ }^{84}$

It is clear from an algebraic standpoint that there is only one condition because $\mu$ enters into only one of Hurwitz's determinants and the others are automatically positive on account of the time constants $\tau$. For a given set of time constants the system will generally be stable when $\mu$ lies below a certain critical value $\mu_{0}$ and unstable for $\mu \geqq \mu_{0}$. When $\mu=\mu_{0}$ there can be one or more oscillations with constant amplitude. In the graphical form of the criterion there is stability when the point $(-1,0)$ lies outside a certain curve traced out by a radius vector representing the complex quantity $\mu Q$ when $p$ is replaced by $i \omega$. The graphical method has been discussed by others. ${ }^{65}$ The differential equation for $x$ can, of course, be replaced by an integral equation which is of the type considered by König or of a

${ }^{63}$ D. G. Prinz, Contributions to the theory of automatic controllers and followers, Journal of Scientific Instruments vol. 21 (1944) pp. 53-64.

${ }^{64} \mathrm{H}$. Nyquist, Regeneration theory, Bell System Technical Journal vol. 11 (1932) pp. 126-147; Annales des Postes, Télégraphes et Téléphones, Paris vol. 23 (1934) pp. 1010-1016. See also K. Kupfmüller, Über die Dynamik der selbsttägigen Verstärkungsregler, Elektrische Nachrichten Technik vol. 5 (1928) pp. 459-467.

${ }_{65}$ R. Feiss, Bestimmung der Regelungsstäbilitat an Hand des Vektorbildes, Zeitschrift für der Verein Deutsches Ingenieures vol. 84 (1940) pp. 819-824. E. Peterson, J. G. Kreer and L. A. Ware, Regeneration theory and experiments, Proceedings of the Institute of Radio Engineers vol. 22 (1934) pp. 1191-1210, Bell System Technical Journal vol. 13 (1934) pp. 680-700. D. G. Reid, Necessary conditions for stability (or self oscillation) of electrical circuits, Wireless Engineers vol. 14 (1937) pp. 588-596. C. A. A. Wass, Feedback amplifiers, Nature vol. 150 (1942) pp. 381-382. 
slightly more general type. In this connection it may be worth while to recall the investigations of $\mathrm{P}$. Hertz ${ }^{66}$ and G. Herglotz ${ }^{67}$ on natural vibrations of an electron. The integral equation considered was then of the form

$$
x(t)=\int_{0}^{\gamma} x(t-T) q(T) d T
$$

and the problem was to find the complex roots of the equation

$$
\int_{0}^{\gamma} e^{-p T} q(T) d T=1 .
$$

The foregoing theory of feedback is based on linear differential equations with constant coefficients and is only approximate. Actually the resistances and capacities may vary with frequency and may even vary with time. In radio-telephony the voice acts so as to modify the resistance of the oscillatory circuit or the capacity of its condenser. J. R. Carson ${ }^{68}$ proposed a differential equation with periodic coefficients as a basis of a theory of modulation and the theory has been worked out more fully by O. Emersleben, ${ }^{69} \mathrm{~W}$. L. Barrow, ${ }^{70}$ and A. Erdélyi. ${ }^{71}$ Conditions of stability are obtained with the aid of the theory of integral equations and of asymptotic solutions of linear differential equations. Feedback is not always desirable. In a discussion of receivers and transmitters for demonstrating frequency modulation M. Hobbs ${ }^{72}$ says that in order to avoid acoustical feedback it is necessary to locate the signal generator and microphone in one studio and the receivers in another.

When the differential equations of the system are nonlinear the theory of stability or of sustained oscillations is more difficult but

${ }^{66} \mathrm{P}$. Hertz, Die Bewegung eines Elektrons unter dem Einflusse einer stets gerichteten Kraft, Math. Ann. vol. 56 (1908) pp. 1-86.

${ }^{67} \mathrm{G}$. Herglotz, Über die Integralgleichungen der Elektronentheorie, ibid. pp. 87-106.

${ }^{68} \mathrm{~J}$. R. Carson, Notes on the theory of modulation, Proceedings of the Institute of Radio Engineers vol. 10 (1922) pp. 57-64.

${ }^{60} \mathrm{O}$. Emersleben, Natural ascillation of circuits containing variable capacities and resistances, Physikalische Zeitschrift vol. 22 (1921) pp. 393-400.

${ }^{70} \mathrm{~W}$. L. Barrow, Frequency modulation and the effects of a periodic capacity variation in a non-dissipative oscillatory circuit, Proceedings of the Institute of Radio Engineers vol. 21 (1933) pp. 1182-1202.

${ }^{71}$ A. Erdélyi, Über die freien Schwingungen in Kondensatorkreisen mit periodisch veranderlicher Kapazitat, Annalen der Physik (5) vol. 19 (1934) pp. 585-622.

${ }^{72} \mathrm{M}$. Hobbs, $A$ low-power transmitter for demonstrating $F-M$ receivers, Electronics vol. 14 (1941) pp. 20-23. 
there is a large literature on the subject. ${ }^{73}$ Nonlinear feedback oscillations have been discussed by G. Hakata and M. Abe. ${ }^{74}$

\subsection{Transcendental equations in the theory of integral equations.} With the abbreviations

$$
(x g y)=\int_{0}^{1} \int_{0}^{1} x(s) g(s, t) y(t) d s d t, \quad(u v)=\int_{0}^{1} u(s) v(s)
$$

a brief study will be made of the linear integral equations

$$
\begin{aligned}
& \text { (1) } f(s)=\int_{0}^{1} g(s, t) F(t) d t+\lambda \int_{0}^{1} h(s, t) F(t) d t+\lambda^{2} \int_{0}^{1} k(s, t) F(t) d t, \\
& \text { (2) } f(s)=F(s)+\lambda \int_{0}^{1} h(s, t) F(t) d t+\lambda^{2} \int_{0}^{1} k(s, t) F(t) d t .
\end{aligned}
$$

When $\lambda$ is a complex quantity $a+i b$ where $a$ and $b$ are real and $f(s)$ is regarded as independent of $\lambda$ and real, the solution $F(t)$ will also be a complex quantity $u(t)+i v(t)$ with $u(t), v(t)$ real provided the kernels $g(s, t), h(s, t), k(s, t)$ are real for real values of $s$ and $t$ which lie between 0 and 1 . The combination $u(t)-i v(t)$ will be denoted by the symbol $F^{*}(t)$ and for both equations the properties of the function

$$
w(\lambda)=\int_{0}^{1} f(s) F(s) d s, \quad w\left(\lambda^{*}\right)=\int_{0}^{1} f(s) F^{*}(s) d s
$$

will be studied. If $c$ is a real constant the zeros and poles of the function $w(\lambda)-c$ will be pseudo-negative when the same is true for the zeros and poles of the function $w\left(\lambda^{*}\right)-c$.

In the important case in which $g(s, t)=g(t, s), h(s, t)=h(t, s)$, $k(s, t)=k(t, s)$ it is readily seen that in the two cases

$$
\begin{aligned}
w\left(\lambda^{*}\right)= & (u g u)+(v g v)+a[(u h u)+(v h v)]+\left(a^{2}-b^{2}\right)[(u k u)+(v k v)] \\
& +i b[(u h u)+(v h v)]+2 i a b[(u k u)+(v k v)], \\
w\left(\lambda^{*}\right)= & (u u)+(v v)+a[(u h u)+(v h v)]+\left(a^{2}-b^{2}\right)[(u k u)+(v k v)] \\
& +i b[(u h u)+(v h v)]+2 i a b[(u k u)+(v k v)] .
\end{aligned}
$$

The right-hand sides of these equations are zero when $\lambda$ is such that $w\left(\lambda^{*}\right)=0$ and also when $\lambda$ is such that $F(t)$ exists when $f(s)=0$. In the important case in which the functions $h$ and $k$ are of positive type

${ }^{73} \mathrm{~K}$. Heegner, The self-oscillating vacuum tube, Arkiv för Elektrot. vol. 9 (1920) pp. 127-152.

${ }^{74}$ G. Hakata and M. Abe, Non-linear differential feedback oscillations, Nippon Electrical Communication Engineering no. 5 (1939) pp. 526-536. 
the integrals $(u h u),(v h v),(u k u),(v k v)$ are all positive and so when the imaginary terms on the right are equated to zero it is seen that either $a$ is negative or $b$ is zero. When $b$ is zero the equation obtained by equating the real part of the right-hand side to zero indicates that $a$ is negative in case $\left(2^{\prime}\right)$ and this is true also in case $\left(1^{\prime}\right)$ if $g(s, t)$ is also of positive type. If $c$ is negative it is readily seen that $a$ must be negative when $w\left(\lambda^{*}\right)=c$.

When $k(s, t)=0$ and $g(s, t), h(s, t)$ are of positive type it is known that the zeros and poles of the function $w(\lambda)$ are all negative and occur alternately. The situation is analogous to that which occurs in the theorem of Routh, Jouguet and Chipart relating to the even and odd parts of an algebraic equation with pseudo-negative roots and so the function $w(\lambda)$ can be used quite often to construct a transcendental equation with only pseudo-negative roots. As an example of the first theorem we take equation (2) with

$$
h(s, t)=p s t, k(s, t)=s(1-t) \text { or } t(1-s)
$$

according as $s \lessgtr t$; the equation for $\lambda$ is then

$$
\operatorname{coth} \lambda=(1 / \lambda)-(1 / p)
$$

when $\lambda$ is a pole. When the equation is written in the form

$$
\operatorname{ch}(\lambda)-(1 / \lambda) \operatorname{sh}(\lambda)+(1 / p) \operatorname{sh}(\lambda)=E(\lambda)+O(\lambda)=0
$$

it is seen that

$$
E\left(z^{1 / 2}\right)=\operatorname{ch}\left(z^{1 / 2}\right)-z^{-1 / 2} \operatorname{sh}\left(z^{1 / 2}\right), \quad z^{-1 / 2} O\left(z^{1 / 2}\right)=z^{-1 / 2} \operatorname{sh}\left(z^{1 / 2}\right) .
$$

It is readily seen that the functions on the right are transcendental functions of $z$ with negative zeros which occur alternately.

In the second theorem if $h(s, t)=s(1-t)$ or $t(1-s)$ according as $s \lessgtr t$ it is found that if

$$
C(z)=\int_{0}^{1} \operatorname{ch}(z t) f(t) d t, \quad S(z)=\int_{0}^{1} \operatorname{sh}(z t) f(t) d t
$$

then the equation

$$
0=\operatorname{sh}(z)+z \operatorname{sh}(z)(f f)-z \operatorname{sh}^{2}(z) S(z) C(z)+\operatorname{sh}(z) \operatorname{ch}(z)[S(z)]^{2}
$$

has only pseudo-negative roots.

\section{The SEPARATION OF VibRations}

4.1. Acoustical filters. The early work of Poisson on the propagation of sound along a branched pipe was followed by the inventions 
of John Herschel and Quincke for the production of interference of waves by the rejunction of the divided branches of a pipe. The theory based on the idea of velocity potential and simplified boundary conditions was improved by Stewart and others and then replaced by a theory of lumped impedances so that the theory of acoustic filters could be developed along the same lines as the theories of mechanical and electrical filters. A good account of the theory from this standpoint is given in the book of Stewart and Lindsay.

The filtering action of a regularly spaced series of similar sheets of muslin was considered by Rayleigh ${ }^{75}$ in the period $1887-1896$ and is described in a passage inserted in the 1896 edition of his Theory of sound. He states that if a moderate number of such sheets be placed parallel to one another and at such distances apart that the partial reflections agree in phase, then a sensitive flame may be powerfully affected. With the aid of a device for adjusting the interval between two consecutive sheets it is easy to find how this interval depends on the wave length $\lambda \cdot$ when the condition for effective reflexion is satisfied. Rayleigh states that with $a=\lambda / 2$ the condition is satisfied for normal incidence but in the actual experiment it is more convenient to use oblique incidence and the calculations necessary for this case are readily made.

In his mathematical investigations ${ }^{78}$ Rayleigh considered the transverse vibrations of a stretched string periodically loaded, but the analysis is rather difficult as it depends on the properties of the solutions of differential equations with periodic coefficients and use is made of infinite determinants as in the work of G. W. Hill. The vibrations of this type of string in which the density varies continuously have been studied further by Strutt ${ }^{77}$ but more progress has been made in the study of the older problem in which the density of the string varies discontinuously. This case will be considered later for both transverse and longitudinal vibrations. The former case is interesting on account of analogies with optical phenomena, the latter on account of the analogies with acoustical phenomena.

The problem of the loaded string was much studied by the great

${ }^{75}$ Lord Rayleigh, Iridescent crystals, Proceedings of the Royal Institute of London vol. 12 (1889) pp. 447-449; Nature vol. 40 (1889) pp. 227-228; Scientific papers, vol. 3, pp. 264-266 (see also pp. 1-14, 204-212); Theory of sound, vol. 2, p. 311.

${ }^{76}$ Lord Rayleigh, On the maintenance of vibrations by forces of double frequency, and on the propagation of waves through a medium endowed with a periodic structure, Philosophical Magazine vol. 24 (1887) pp. 145-159; On the remarkable phenomenon of crystalline reflexion described by Stokes, ibid. vol. 26 (1888) pp. 256-265.

${ }^{77}$ M. J. O. Strutt, Eigenschwingungen einer Saite mit sinusformiger Massenverteilung, Annalen der Physik vol. 85 (1928) pp. 129-136. 
mathematicians of the eighteenth century and many of their results are given by Routh in his Rigid dynamics. Routh also mentions that in April 1875 Lord Kelvin studied the vibrations and waves in a stretched uniform chain of symmetrical gyrostats connected together by universal flexure joints. His thoughts returned to this subject in his Baltimore lectures of 1884 .

The theory of the loaded string became definitely associated with the theory of mechanical filters in 1898 when Godfrey and Lamb published their researches and when Campbell and Pupin became interested in the properties of the loaded electrical transmission line. Acoustic filters are much used as mufflers for internal combustion engines and as may be seen from the lists of patents in the Journal of the Acoustical Society of America baffles, holes and side branches in the exhaust pipe are among the devices used. Resonating side branches sometimes communicate with one another through partitions of absorbing material. Even a long pipe has a filtering action as the attentuation is higher for sound of some frequencies than for sound of some other ranges of frequency. Problems relating to pipes will be discussed in the section dealing with hydrodynamics in which some consideration will be devoted not only to the elimination of noise but also the reduction of dangerous vibrations in hydraulic pipe lines.

4.2. Passage of sound through a slab. Let $\rho, v$, and $Z=\rho v$ be the density velocity of sound and radiation resistance of a homogeneous slab of thickness $a$ which is of infinite extent in any direction parallel to the plane faces. Let $\rho, v^{\prime}, Z^{\prime}=\rho^{\prime} v^{\prime}$ be the corresponding quantities for the medium outside the slab. For normal incidence of waves on the face $x=0$ the velocity potentials are

$$
\begin{aligned}
& \phi=D e^{i w\left(t-x / v^{\prime}\right)}+E e^{i w\left(t+x / v^{\prime}\right)} \quad \text { for } x \leqq 0, \\
& \phi=B e^{i w(t-x / v)}+C e^{i w(t+x / v)} \quad \text { for } 0 \leqq x \leqq a \text {, } \\
& \phi=A e^{i w\left(t-x / v^{\prime}\right)} \quad \text { for } x \geqq a .
\end{aligned}
$$

The boundary conditions are

$$
\begin{gathered}
\rho^{\prime}(D+E)=\rho(B+C), \quad \rho\left(B e^{-i s}+C e^{i s}\right)=\rho^{\prime} A e^{-i s^{\prime}}, \\
i s^{\prime}(E-D)=i s(C-B), \quad i s\left(C e^{i s}-B e^{-i s}\right)=-i s^{\prime} A e^{-i s^{\prime}}
\end{gathered}
$$

where $s=w a / v, s^{\prime}=w a / v^{\prime}$. Thus

$$
A / D=2 e^{i s} /\left[2 \cos (s)+i\left(Z / Z^{\prime}+Z^{\prime} / Z\right) \sin (s)\right]
$$

and the coefficient of reflection is 


$$
r=\left(Z^{\prime} / Z-Z / Z^{\prime}\right)\left[4 \cot ^{2}(s)+\left(Z^{\prime} / Z-Z / Z^{\prime}\right)^{2}\right]^{-1 / 2} .
$$

This is the formula of Lord Rayleigh. ${ }^{78}$ Interesting applications of this formula to the reflection and transmission of sound through partitions have been made by Boyle ${ }^{69}$ and Davis. ${ }^{80} \mathrm{It}$ is clear that $r=0$ when $\sin (s)=0$ and so there are certain critical thicknesses $a$ for which there is no reflection of sound waves of the prescribed frequency $w / 2 \pi$. Davis regards the formula as applicable to the transmission of sound through light thin panels such as sheets of paper, sailcloth or fibre board. When $a$ is very small there is a tendency for the reduction factor of the energy to vary as the square of the frequency $f$ of the incident sound. With materials as light as paper a term due to air damping is important and there is less variation with $f$. For heavy panels such as two inch boards or brick walls the reduction factor is less than that given by Rayleigh's formula. Davis has given a formula

$$
\text { Reduction factor }=(1 / 2 R)^{2}\left[(r+2 R)^{2}+(m / w)^{2}\left(w^{2}-w_{0}^{2}\right)\right]
$$

which indicates that resonances can account for a reduced insulating value. This formula is derived from a differential equation of type

$$
\ddot{m \xi}+(r+2 R) \dot{\xi}+S \xi=2 R \xi_{0} e^{i \omega t}, \quad w_{0}=(S / m)^{1 / 2} .
$$

To account for the behavior of actual panels it seems necessary to assume that there are several modes of vibration with which there can be resonance. This is in accordance with the general theory of the vibration of plates and with experiment as is pointed out by Davis and Littler. ${ }^{81}$

In the work of Boyle on the influence of the thickness of the plate on the transmission of sound, use was made of a high $f(135000 \sim$, $300000 \sim$ and $528000 \sim)$. It was found that when $a$ was a large multiple of $\lambda / 4$ (where $\lambda$ is the wave length in the plate) there was a maximum of energy reflected and a minimum of energy transmitted. When $a$ was a few integral multiples of $\lambda / 2$ there was an almost complete transmission of energy. Thus in the latter case the plate acted as a

${ }^{78}$ Lord Rayleigh, Theory of sound, vol. 2, 1896, p. 88.

${ }^{79} \mathrm{R}$. W. Boyle, Transmission of sonic and ultrasonic waves through partitions, Nature vol. 121 (1928) pp. 55-56. See also R. W. Boyle and D. K. Froman, Canadian Journal of Research vol. 1 (1929) pp. 405-424.

${ }^{80}$ A. H. Davis, Transmission of sound through partitions, Philospohical Magazine (7) vol. 15 (1933) pp. 309-316.

${ }^{81}$ A. H. Davis and T. S. Littler, The measurement of transmission of sound by partitions of various materials, Philosophical Magazine (7) vol. 3 (1927) pp. 177-194, vol. 7 (1929) pp. 1050-1062. 
high pass filter and in the former case as a high frequency rejector. This work has been continued by Boyle and Sproule ${ }^{82}$ with the aid of a torsion pendulum and the previous conclusion confirmed.

4.3. Multiple partitions. The passage of sound through several slabs of different materials is of some interest but the analysis is rather complicated. The case of three different media was considered by Brillie and his work is discussed by Stewart and Lindsay. The case of five media two pairs of which are alike in properties and are symmetrically related to a third medium in the middle is of much interest in relation to the sandwich type of radiator and receiver which was used at one time in underwater work. The natural vibrations of such a sandwich are of interest.

The vibrations of a column of gas, one portion of which is at a temperature $T_{1}$ and the other at a temperature $T_{2}$ have been studied by Lees $^{83}$ in connection with some experiments on the vibration of travelling flames made by Coward and Hartwell. ${ }^{84}$ Lees considered 3 cases: $1^{\circ}$. Tube closed at both ends. $2^{\circ}$. Tube open at both ends. $3^{\circ}$. Tube closed at one end and open at the other. In the last named case the frequency of vibration is determined by the equation

$$
\left(n_{1} / F_{1}\right) \tan \left(m_{1} \pi / 2\right)=-\left(n_{2} / F_{2}\right) \tan \left[\left(m_{2}-1\right)(\pi / 2)\right]
$$

where $n_{1}$ is the fundamental frequency when the whole column is at temperature $T_{1}, n_{2}$ is the corresponding frequency when the temperature is $T_{2}, F_{1}$ and $F_{2}$ are the moduli of adiabatic elasticity for longitudinal displacements in the column at the two temperatures and

$$
m_{1}=n a / n_{1} c, \quad m_{2}=n b / n_{2} c, \quad a+b=c
$$

where $a, b$ are the lengths of the two portions of the tube.

Meyer observes that it is well known that the sound-damping action of a homogeneous wall increases with its thickness provided the exciting frequency is sufficiently higher than the natural frequency of vibration of the wall-a condition that is usually satisfied. When several such walls have air between them the composite wall acts as a mechanical damper because the layers of air form buffers if they are small compared with the wave length. If $m$ denotes the mass of the

${ }^{82}$ R. W. Boyle and D. O. Sproule, Transmission of sound energy and thickness of plate transmitter at normal incidence-ultrasonic method, Canadian Journal of Research vol. 2 (1930) pp. 3-12.

${ }^{83}$ C. H. Lees, Free periods of a composite elastic column or composite stretched wire, Proceedings of the Physical Society of London vol. 41 (1929) pp. 204-213.

${ }_{84} \mathrm{~J}$. F. Coward and F. J. Hartwell, Extinction of methane flames by diluent gases, Journal of the Chemical Society vol. 129 (1926) pp. 1522-1532. 
wall in grams per square centimeter, if $l$ is the length of an air buffer, if $v$ is the velocity of sound and if $\rho$ is the density of the air, the fundamental natural frequency is

$$
f_{0}=(v / \pi)(\rho l / m)^{1 / 2} .
$$

This formula has been confirmed over a frequency range from $400 \sim$ to $7000 \sim$. Meyer ${ }^{85}$ states that in such composite walls the velocity of transmission decreases as the frequency increases but the damping does not seem to increase very rapidly. Investigations led to the following rules for the design of composite walls. The dimensions should be such that $f_{0}$ is less than the practically important range of $f$ 's and the cross vibrations in the air buffers should be damped. By following these rules sound can be effectively damped without the use of heavy walls. A four-fold composite wall forty centimeters thick (153/4 inches) weighing 50 kilograms per square meter ( 10.25 pounds per square foot) gave better insulation than a solid brick wall weighing $1000 \mathrm{~kg}$. per sq.m. The effect of composite walls has been discussed by many other writers ${ }^{86}$ and the mathematical theory has been elucidated by Constable. ${ }^{87}$ For $f>f_{0}$ insulation is at first decreased as the separation of the walls is increased, but it afterwards increases continuously up to a point at which the separation is approximately $\lambda / 4$. After this point it decreases to a second minimum and thereafter minima occur at successive increases of $\lambda / 2$ in the separation of the walls. The first minimum at which the insulation can be less than that of one component alone can be attributed to the effect of air coupling.

The properties of a double partition constructed from dissimilar components were examined by Renault ${ }^{88}$ and by Constable. ${ }^{87}$ The latter found that at $f$ 's for which the resonances of the components

${ }^{85}$ E. Meyer, Die Mehrfachwand als akustisch-mechanische Drosselkette, Zeitschrift für Technische Physik vol. 16 (1936) pp. 565-566; Über das Schallschluckvermögen schwingungsfähiger, nichtpöroser Stoffe, Elektrische Nachrichten Technik vol. 13 (1936) pp. 95-102.

${ }^{86} \mathrm{E}$. Wintergast, Theorie der Schalldurchlassigkeit von einfachen und zusammengesetzten Wänden, Schalltechnik vol. 4 (1931) pp. 85-91, vol. 5 (1932) pp. 1-8. J. E. R. Constable and G. H. Aston, The sound insulation of single and complex partitions, Philosophical Magazine (7) vol. 23 (1937) pp. 161-181. E. Lubcke and A. Eisenberg, Zur Schallübertragung von dïnnen Einfachwänden, Zeitschrift Technische Physik vol. 18 (1937) pp. 170-174.

$87 \mathrm{~J}$. E. R. Constable, The acoustical insulation afforded by double partition constructed from similar components, Philosophical Magazine (7) vol. 18 (1934) pp. 321343; Acoustical insulation afforded by double partitions constructed from dissimilar components, ibid. vol. 26 (1938) pp. 253-259.

${ }^{88} \mathrm{~L}$. Renault, La transmission $d u$ son a travers cloisons métalliques, Revue d'Acoustique vol. 6 (1937) pp. 69-101. 
and the air coupling resonance can be neglected, the insulation to be obtained from a double partition for a given total weight and thickness is greatest when the components are similar.

The transmission of sound by a series of equidistant similar partitions has been studied by Hurst ${ }^{89}$ whose results are very similar to those of Lamb in his first example of a mechanical filter ( $\$ 4.5)$. The method of indicating the regions of attenuation is also similar to that of Lamb. Hurst gives also a theory of transmission through a series of circular panels each of which is set into a rigid wall. In this theory effects of diffraction are taken into account.

4.4. Results found by energy methods. The absorption of sound by a porous wall was studied theoretically by Rayleigh with the aid of a theory of the propagation of sound in a capillary tube which will be examined in $\$ 6.89 \mathrm{a}$ There are, however, some results which can be obtained by energy methods which may be mentioned here for comparison with the other results.

4.4a. The passage of energy through a single absorbing wall. By means of an extension of the analysis of Buckingham and Eckhardt, 90 Davis $^{91}$ has obtained the following equations for two rooms separated by an absorbing wall:

$$
4 V \dot{I}+v a S I=4 E+v k W I, \quad 4 V_{1} \dot{I}_{1}+v a_{1} S_{1} I_{1}=v k W I .
$$

The first equation refers to the room containing the source of sound which is supposed to emit energy at a constant rate $E$ while operative. $W$ denotes the area of the wall used as a partition between the two rooms. $V, V_{1}$ are the volumes of the rooms; $S, S_{1}$ are the respective total areas of the exposed surfaces of the walls of these rooms (including the partition); $a, a_{1}$ are the mean fractions of incident energy lost by the respective rooms at each reflection by absorption or transmission to other rooms; $v$ is the velocity of sound and $k$ is a factor of type $a$ for the partition. In the steady state $I=I_{1}=0$ so the maximum values of $I$ and $I_{1}$ are $J$ and $J_{1}$ respectively, where

${ }^{89}$ D. G. Hurst, The transmission of sound by a series of equidistant partitions, Canadian Journal of Research vol. 12 (1935) pp. 398-407.

${ }^{89 a}$ References to $\$ \$ 5$ and 6 refer to material which the author hopes will appear after the war.

${ }^{90} \mathrm{E}$. A. Eckhardt, The acoustics of rooms, reverberation, Journal of the Franklin Institute vol. 195 (1923) pp. 799-814.

${ }^{21} \mathrm{~A}$. H. Davis, Reverberation equation for two adjacent rooms connected by an incompletely sound-proof partition, Philosophical Magazine (6) vol. 50 (1925) pp. 75-80. See also C. F. Eyring, Methods of calculating the average coefficient of sound absorption, Journal of the Acoustical Society vol. 4 (1933) pp. 178-192. 


$$
J=\frac{4 E a_{1} S_{1}}{v\left(a S a_{1} S_{1}-k^{2} W^{2}\right)}, \quad J_{1}=J k W /\left(a_{1} S_{1}\right) .
$$

At time $t$ after the source has been cut off

$$
\begin{aligned}
& \frac{I}{J}=\frac{\lambda_{2}-\lambda_{0}}{\lambda_{2}-\lambda_{1}} e^{-\lambda_{1} t}+\frac{\lambda_{1}-\lambda_{0}}{\lambda_{1}-\lambda_{2}} e^{-\lambda_{2} t}, \\
& \frac{I_{1}}{J_{1}}=\frac{\lambda_{2}}{\lambda_{2}-\lambda_{1}} e^{-\lambda_{1} t}+\frac{\lambda_{1}}{\lambda_{1}-\lambda_{2}} e^{-\lambda_{2} t}
\end{aligned}
$$

where

$$
\begin{aligned}
\left.\begin{array}{c}
\lambda_{1} \\
\lambda_{2}
\end{array}\right\} & =(v / 8)\left\{N+N_{1} \mp\left[\left(N_{1}-N\right)^{2}+4 k^{2} W^{2} / V V_{1}\right]^{1 / 2}\right\}, \\
N & =a S / V, \quad N_{1}=a_{1} S_{1} / V_{1}, \quad \lambda_{0}=4 V_{1} \lambda_{1} \lambda_{2} /\left(v a_{1} S_{1}\right) .
\end{aligned}
$$

$4.4 \mathrm{~b}$. The passage of waves through a series of interceptors. The reflection of light from a pile of plates was discussed in a restricted form by Augustin Fresnel ${ }^{92}$ and Franz Neumann, the formulae of the latter being quoted by Wild in 1856. In 1862 Stokes $^{93}$ treated the problem in a general way by means of the functional equations

$$
\begin{aligned}
r(m+n) & =r(m)+r(n)[t(m)]^{2} /[1-r(m) r(n)], \\
t(m+n) & =t(m) t(n) /[1-r(m) r(n)]
\end{aligned}
$$

in which $r(m)$ denotes the fraction of energy reflected from a pile of $m$ plates, $t(m)$ denotes the fraction of energy transmitted and $a(m)=1-r(m)-t(m)$ is the fraction of energy absorbed in the plates. In the derivation of these equations the plates are supposed to be all formed of the same material and to be all of the same thickness. The plates themselves and the interposed sheets of air are supposed to be so thick that the phenomenon of the colours of thin plates does not occur to any appreciable extent. The analysis deals only with intensities of light or other wave motion.

If $r(1)=r, t(1)=t$ and quantities $a, b$ are defined by the equations

$$
r \operatorname{ch} a+t \operatorname{ch} b=1, \quad r \operatorname{sh} a-t \operatorname{sh} b=0,
$$

${ }^{92} \mathrm{~A}$. Fresnel, Calculs sur les intensitês de lumière rêfléchi par une, deux et quatre glacés, Oeuvres complètes, vol. 2, pp. 789-792.

${ }^{93} \mathrm{Sir}$ George Stokes, On the intensity of the light reflected from or transmitted through a pile of plates, Proc. Roy. Soc. London vol. 11 (1862) pp. 545-556. J. Stirling, Note on a functional equation treated by Sir George Stokes, Proc. Roy. Soc. London Ser. A vol. 90 (1914) pp. 237-239. 
an appropriate solution is

$$
r(n)=\frac{\operatorname{sh}(n b)}{\operatorname{sh}(a+n b)}, \quad t(n)=\frac{\operatorname{sh}(a)}{\operatorname{sh}(a+n b)} .
$$

It should be noticed that

$4 \operatorname{sh}^{2}(a / 2)=\left(r^{-1 / 2}-r^{1 / 2}\right)^{2}-t^{2} / r, \quad 4 \operatorname{sh}^{2}(b / 2)=\left(t^{-1 / 2}-t^{2}\right)^{2}-r^{2} / t$.

Since $1-r>t$ and $1-t>r$ the quantities $a$ and $b$ defined by these equations are both real. It should be noticed also that

$$
\begin{gathered}
t^{2}=1-2 r \operatorname{ch} a+r^{2}, \quad r^{2}=1-2 t \operatorname{ch} b+t^{2}, \\
{[t(n)]^{2}=1-2 r(n) \operatorname{ch} a+[r(n)]^{2},} \\
{[r(n)]^{2}=1-2 t(n) \operatorname{ch}(n b)+[t(n)]^{2} .}
\end{gathered}
$$

Hence in order to investigate the effect of frequency on $r(n)$ and $t(n)$ it is useful to have plots in the $r t$-plane of the two systems of hyperbolas $a=$ constant, $b=$ constant.

4.5. Mechanical filters. A general theory of mechanical filters was given by Horace Lamb ${ }^{94}$ and Charles Godfrey ${ }^{95}$ in 1898. In the analysis of Lamb dynamical systems of any degree of complexity but all exactly alike are supposed to be interpolated at regular intervals along a line which is regarded for simplicity as an infinite string capable of longitudinal vibrations. The position and configuration of any one of these systems is imagined to be determined by the coordinate $\xi$ of the point of the string where it is attached and by means of $n$ other coordinates $q_{8}(s=1,2, \cdots, n)$. The kinetic energy $T$ and potential energy $V$ are, moreover, assumed to have the forms

$$
2 T=\sum_{r=1}^{n} a_{r} \dot{q}_{r}^{2}+2 \sum_{r=1}^{n} \alpha_{r} \dot{\xi} \dot{q}_{r}+P \xi^{2}, \quad 2 V=\sum_{r=1}^{n} b_{r} q_{r}^{2}+2 \sum_{r=1}^{n} \beta \xi q_{r}+Q \xi^{2},
$$

so that the equations of motion are

$$
\begin{aligned}
a_{r} \ddot{q}_{r}+b_{r} q_{r}+\alpha_{r} \ddot{\xi}+\beta_{r} \xi & =0, \quad r=1,2, \cdots, n, \\
P \ddot{\xi}+Q \xi+\sum_{r=1}^{n}\left(\alpha_{r} \ddot{q}_{r}+\beta_{r} q_{r}\right) & =X
\end{aligned}
$$

where $X$ is the external force corresponding to the coordinate $x$ repre-

${ }^{94} \mathrm{H}$. Lamb, On waves in a medium having a periodic discontinuity of structure, Memoirs of the Manchester Literary and Philosophical Society vol. 42, no. 3, 1898, 20 pp.

${ }_{95} \mathrm{C}$. Godfrey, Discontinuities of wave-motion along a periodically loaded string, Philosophical Magazine (5) vol. 45 (1898) pp. 356-363. 
senting in Lamb's model the difference of tensions on the two sides of the gap into which the dynamical system is introduced.

When all quantities contain the time factor $\exp (i k c t)$ the first $n$ equations of motion give the relations

$$
q_{r}=-\frac{k^{2} c^{2} \alpha_{r}-\beta_{r}}{k^{2} c^{2} a_{r}-b_{r}}, \quad r=1,2, \cdots, n,
$$

and the last equation gives

$$
\left\{k^{2} c^{2} P-Q-\sum_{r=1}^{n}\left(k^{2} c^{2} \alpha_{r}-\beta_{r}\right)^{2} /\left(k^{2} c^{2} a_{r}-b_{r}\right)\right\} \xi=-X .
$$

The successive dynamical systems may be distinguished by suffixes $\left(D_{r}\right)$ and may be pictured as particles at intervals of length $a$. If $2 \pi / k$ is the wave length for a disturbance with the same time factor on the unloaded string, the motion to the right of $D_{r}$ is of type

$$
\xi=\xi_{r} \cos (k x)+\left[\xi_{r+1}-\xi_{r} \cos (k a)\right] \sin (k x) \csc (k a)
$$

while on the left it is

$$
\xi=\xi_{r} \cos (k x)+\left[\xi_{r} \cos (k a)-\xi_{r-1}\right] \sin (k x) \csc (k a) .
$$

The tensions of the string on the two sides of $D_{r}$ are

$$
\begin{aligned}
& E(d \xi / d x)_{+}=k E \csc (k a)\left[\xi_{r+1}-\xi_{r} \cos (k a)\right], \\
& E(d \xi / d x)_{-}=k E \csc (k a)\left[\xi_{r} \cos (k a)-\xi_{r-1}\right],
\end{aligned}
$$

so

$$
X_{r}=k E \csc (k a)\left[\xi_{r+1}-2 \xi_{r} \cos (k a)+\xi_{r-1}\right]
$$

and there is a difference equation

$$
\xi_{r+1}-2[\cos (k a)-f(k) \sin (k a)] \xi_{r}+\xi_{r-1}=0
$$

where

$$
2 k E \cdot f(k)=k^{2} c^{2} P-Q-\sum_{r=1}^{n} \frac{\left(k^{2} c^{2} \alpha_{r}-\beta_{r}\right)^{2}}{k^{2} c^{2} a_{r}-b_{r}} .
$$

The solution of the difference equation has different forms according as the coefficient of $2 \xi_{r}$ does or does not lie between the limits \pm 1 . The critical values of $k$ are determined by

$$
C(k a) \equiv \cos (k a)-f(k) \sin (k a)= \pm 1 .
$$

The roots of this equation give the ranges of frequency within which there is total reflection or partial transmission. 
When $C(k a)$ lies between \pm 1 there is a real angle $\theta$ such that

$$
\cos \theta=C(a k)
$$

and then the solution of the difference equation is

$$
\xi_{r}=P e^{i r \theta}+Q e^{-i r \theta}
$$

where $P$ and $Q$ are suitable constants independent of $r$. The wavelength $\lambda$ in the loaded medium may be defined to be

$$
\lambda=(2 \pi a / \theta)=(k a / \theta) \lambda_{0}
$$

where $\lambda_{0}=(2 \pi / k)$ is the wave length in the unloaded medium. The wave velocity in the loaded medium may also be regarded as given by the equation

$$
v=(\lambda k c / 2 \pi) .
$$

The group velocity is then

$$
w=v-\lambda d v / d \lambda=-\left(\lambda^{2} c / 2 \pi a\right)[d(k a) / d \theta](d \theta / d \lambda)=-\left[c / C^{\prime}(a k)\right] \sin \theta .
$$

Unless $C^{\prime}(a k)=0$ for the critical values of $k$ the group velocity will be zero when $k$ has a critical value and $\sin \theta=0$. When $\theta$ lies between 0 and $\pi$ the group velocity is positive when $C^{\prime}(a k)$ is negative. The behavior of $v$ may be found by Lamb's graphical method in which use is made of the curves

$$
y=\cot (x / 2), \quad y=-\tan (x / 2), \quad y=f(x / a),
$$

and the points of intersection of the last curve with the first two. With many forms of the function $f$ the intervals of partial transmission are represented by intervals beginning respectively at $\pi, 2 \pi, 3 \pi, \cdots$, and the intervals of total reflection by intervals ending at these points. There may also be an interval of partial transmission between 0 and $\pi$.

In Lamb's first example $f(k)=\mu k a / 2$, where $\mu=M / \rho a$. The dynamical system then consists of a mass $M$ and $\rho a$ is the mass of the portion of the string between two consecutive masses. When the string is infinite in both directions there is transmission only for certain values of $k$ below a certain limit. When $k a$ is large $\theta$ is given approximately by $\theta^{2}=k^{2} a^{2}(1+\mu)$ and the refractive index $N$ is

$$
N=\left(\lambda / \lambda_{0}\right)=(1+\mu)^{1 / 2} .
$$

The effect of the loads is to increase the average density of the medium.

In Lamb's second example the mass $M$ is urged towards its mean position by a spring and so 


$$
f(k)=\frac{\mu}{2}\left(k a-\frac{\sigma^{2} a^{2}}{c^{2} k a}\right) .
$$

The chief difference between this case and the last is that there is an interval of total reflection beginning at $k a=0$ which is separated by an interval of partial transmission from the interval of total reflection which ends at $k a=\pi$. Lamb regards this as remarkable.

In Lamb's third example $f(k)=(\mu k a / 2)\left(1-k^{2} c^{2} / \sigma^{2}\right)$. The chief difference between this case and the first is that as the frequency increases the intervals of partial transmission become wider and wider instead of shorter and shorter so that the medium is transparent for short waves of high frequency. This example was offered by Lamb to illustrate the transparency of a medium to Röntgen rays, a phenomenon which Stokes ${ }^{96}$ had endeavoured to explain in his Wilde lecture of 1897.

In Lamb's model there are a number of equal light rigid circular frames each of which is attached to the string at opposite ends of a diameter and has in its interior a particle $M$ connected with the frame by means of similar springs so that the particle is at the center of the frame when the springs are equally extended. If $\xi_{\text {s }}$ denotes the displacement of the point of the string to which one of the frames is attached and if $\eta_{\mathrm{s}}$ denotes the displacement of the associated particle, the equation of motion of this particle is

$$
M \ddot{\eta}_{s}+M \sigma^{2}\left(\eta_{s}-\xi_{s}\right)=0
$$

while there is a balance of tensions if

$$
\begin{aligned}
0= & k E\left[\xi_{s+1}-\xi_{s} \cos (k a)\right]-k E\left[\xi_{s} \cos (k a)-\xi_{s-1}\right] \\
& +M \sigma^{2}\left(\eta_{s}-\xi_{s}\right) \csc (k a) .
\end{aligned}
$$

These equations give the relation between $\xi_{8+1}, \xi_{8}$ and $\xi_{8-1}$ of the type mentioned.

Lamb's model furnishes a particular type of high pass filter.

4.6. Selective reflection. In Lamb's work the string is unloaded to the left of 0 and on the right has masses $M$ at the points $x=0, a, \cdots, n a$ and is unloaded beyond the point $n a$. For $x<0$ it is assumed that

$$
\xi=e^{i k(c t-x)}+A e^{i k(c t+x)} .
$$

${ }^{96} \mathrm{Sir}$ George Stokes, On the nature of the Röntgen rays, Memoirs of the Manchester Literary and Philosophical Society vol. 41, no. 15, 1897; Mathematical and physical papers, vol. 5, pp. 256-277. 
For $0<x<n a$ it is assumed that

$$
\xi_{r}=C e^{i(k c t-r \theta)}+D e^{i(k c t+r \theta)}
$$

and for $x>n a$

$$
\xi=B e^{i k(c t-x)} \text {. }
$$

The kinematical conditions at $x=0$ and $x=n a$ are

$$
1+A=C+D, \quad B e^{-i n k a}=C e^{-i n \theta}+D e^{i n \theta}
$$

while the dynamical equations are

$$
\begin{aligned}
1-A= & C i \csc (k a)\left[\cos (k a)-e^{i \theta}\right]+i D \csc (k a)\left[\cos (k a)-e^{-i \theta}\right], \\
B e^{-i n k a}= & i C e^{-i n} \csc (k a)\left[e^{-i \theta}-\cos (k a)\right] \\
& +i D e^{i n \theta} \csc (k a)\left[e^{i \theta}-\cos (k a)\right]
\end{aligned}
$$

and from these equations it is found that

$$
\begin{aligned}
& B=\frac{\sin \theta \sin (k a) \exp [(n+1) i k a]}{\sin \theta \sin (k a) \cos (n+1) \theta+i[1-\cos \theta \cos (k a)] \sin (n+1) \theta}, \\
& A=\frac{i(\cos \theta-\cos (k a)) \sin (n+1) \theta e^{i k a}}{\sin \theta \sin (k a) \cos (n+1) \theta+i[1-\cos \theta \cos (k a)] \sin (n+1) \theta} .
\end{aligned}
$$

The intensities of the reflected and transmitted waves are respectively

$$
\begin{aligned}
& R=\left[(\cos \theta-\cos (k a))^{2} \sin ^{2}(n+1) \theta\right] / H, \\
& T=\left[\sin ^{2} \theta \sin ^{2}(k a)\right] / H,
\end{aligned}
$$

where $H=\sin ^{2} \theta \sin ^{2}(k a) \cos ^{2}(n+1) \theta+(1-\cos \theta \cos (k a))^{2} \sin ^{2}(n+1) \theta$. When $n$ is large slight changes in $k a$ and $\theta$ will make $\cos (n+1) \theta$ and $\sin (n+1) \theta$ vary considerably. Mean values of $R$ and $T$ for values of $\lambda$ close to $2 \pi / k$ may be found by integration as in Kirchhoff's Optik (p. 165) and in Lamb's paper by using the well known formula

$$
\int_{0}^{\pi / 2} \frac{d \phi}{\alpha^{2} \cos ^{2} \phi+\beta^{2} \sin ^{2} \phi}=\pi / 2 \alpha \beta .
$$

Consequently

$$
\begin{array}{ll}
\bar{T}=\left|\frac{\sin \theta \sin (k a)}{1-\cos \theta \cos (k a)}\right|, & \bar{R}=1-\bar{T}, \\
\bar{T}=\frac{1-q^{2}}{1+q^{2}}, & \bar{R}=\frac{2 q^{2}}{1+q^{2}}
\end{array}
$$

where 


$$
q=\left|\frac{\sin (k a / 2-\theta / 2)}{\sin (k a / 2+\theta / 2)}\right| .
$$

This quantity $q$ is the coefficient of reflection obtained by Lamb in his analysis of the case in which waves travelling towards 0 along the unweighted portion of the string are reflected from the weighted portion on which weights evenly spaced extend to infinity. This case is similar to the problem considered by Godfrey.

An interesting variation of the present problem is one in which the unweighted portions of the string are replaced by weighted portions in which the weights are evenly spaced but at intervals differing in length from the intervals in the intermediate portion of the string. If the intervals in this intermediate portion are greater than in the rest of the string the problem is analogous to that of waves passing from a dense medium into a plate composed of a light substance. This problem is of interest for both longitudinal and transverse vibrations.

4.7. The reactance theorem and its generalizations. In 1886 the late Lord Rayleigh ${ }^{97}$ gave a dynamical theorem which in modern terminology is a theorem relating to the driving point impedance in a chain like dynamical system. Applications to electrical networks were considered. In 1908 the theorem was extended by the present author $^{98}$ to a linear integral equation

$$
u(s)=U(s)-\lambda \int_{0}^{1} k(s, t) U(t) d t
$$

in which $k(s, t)$ is a real symmetric kernel. If

$$
w(\lambda)=\int_{0}^{1} u(s) U(s) d s
$$

the theorem then states that the zeros and poles of the function $w(\lambda)$ are all positive when the kernel is such that for any real nonvanishing function $x(s)$ the double integral

$$
(x k x)=\int_{0}^{1} \int_{0}^{1} x(s) k(s, t) x(t) d s d t
$$

${ }^{97}$ Lord Rayleigh, The reaction upon the driving-point of a system executing forced harmonic oscillations of various periods, with applications to electricity, Philosophical Magazine (5) vol. 21 (1886) pp. 369-381; Scientific papers, vol. 2, pp. 475-485.

${ }_{98} \mathrm{H}$. Bateman, The reality of the roots of certain transcendental equations occurring in the theory of integral equations, Trans. Cambridge Philos. Soc. vol. 20 (1908) pp. 371382; Notes on integral equations III, the homogeneous integral equation of the first kind, Messenger of Mathematics vol. 39 (1909) pp. 6-19. 
is positive. The derivative $w^{\prime}(\lambda)$ is then positive for all real values of $\lambda$, becoming infinite, if at all, only at certain values of $\lambda$ at which the homogeneous integral equation (with $u=0$ ) can have a nonvanishing solution.

It is the analogue of this theorem for a system of linear algebraic equations

$$
u_{r}=U_{r}-\lambda \sum_{s=1}^{n} k_{r, s} U_{s}, \quad r=1,2, \cdots, n,
$$

which is essentially the theorem given by Rayleigh. The real constants $k_{r, s}$ are then supposed to be symmetric $\left(k_{r, s}=k_{s, r}\right)$ and such that the quadratic form

$$
(x k x)=\sum_{r, s=1}^{n} x_{r} k_{r, s} x_{s}
$$

is positive for all nonvanishing sets of real quantities $x_{r}$. The function $w(\lambda)$ is then defined by the equation

$$
w(\lambda)=\sum_{r=1}^{n} u_{r} U_{r}
$$

and its derivative is positive for all real values of $\lambda$. This indicates that the zeros and poles of the rational function $w(\lambda)$ occur alternately.

In $1922 \mathrm{G}$. A. Campbell ${ }^{19}$ gave an expression for the driving-point impedance $Z$ of a non-dissipative reactance network

$$
Z=R+i X=i M \frac{f\left(f_{2}^{2}-f^{2}\right) \cdots\left(f_{2 n}^{2}-f^{2}\right)^{m}}{\left(f_{1}^{2}-f^{2}\right) \cdots\left(f_{2 n-1}^{2}-f^{2}\right)}
$$

in which $M$ is a positive constant, $f_{2}, f_{4}, \cdots$ are constants representing resonant frequencies, $f_{1}, f_{3}, \cdots$ are constants representing antiresonant frequencies and the exponent $m$ of the last factor in the numerator is 1 or 0 according as a resonant or anti-resonant frequency is the last member when the frequencies $f_{r}$ are arranged in order of increasing magnitude

$$
0 \leqq f_{1}<f_{2}<f_{3}<f_{4} \cdots .
$$

This type of arrangement is possible because $d X / d f>0$ and so the zeros and poles of $X$ occur alternately. The theorem may be derived from the previous theorem for a system of algebraic equations by

${ }^{{ }^{9}}$ G. A. Campbell, Physical theory of the electric wave-filter, Bell System Technical Journal vol. 1 (1922) pp. 30-31. 
taking all but one of the quantities $u_{r}$ to be zero. There is a corresponding theorem for the more general set of linear equations

$$
u_{r}=\sum_{s=1}^{n} h_{r, s} U_{s}-\lambda \sum_{s=1}^{n} k_{r, s} U_{s}, \quad r=1,2, \cdots, n,
$$

where the coefficients $h_{r, s}, k_{r, s}$ are real symmetric quantities such that the quadratic forms $(x h x),(x k x)$ are positive for nonvanishing sets of quantities $x_{r}$. There is also a similar theorem for the integral equation of the first kind

$$
u(s)=\int_{0}^{1} h(s, t) U(t) d t-\lambda \int_{0}^{1} k(s, t) U(t) d t
$$

in which $h(s, t), k(s, t)$ are real symmetric kernels such that $(x h x)$ and $(x k x)$ are positive for any nonvanishing real continuous function $x\left(z^{\prime}\right)$. In all these theorems the function $w(\lambda)$ increases continually with $\lambda$ but if the conditions imposed on the function $k$ or the coefficients $k_{r, s}$ are relaxed so that $k$ is merely real and symmetric then the function $\lambda w(\lambda)$ increases with $\lambda$ and there are zeros and poles of this function which occur alternately but are not all positive. The theorem that $w(\lambda)$ or $\lambda w(\lambda)$ increases with $\lambda$ may be proved in many ways but one way is to use partial fractions. A number of theorems may be proved in this way, some of them are well known in the theory of algebraic equations and some have found useful applications in the theory of electric filters.

The reactance theorem has been proved by many writers. Zobel ${ }^{100}$ gives a proof by induction while Foster ${ }^{101}$ bases a proof on dynamical theorems of Routh, Rayleigh and Webster. Foster also calls attention to a connection with algebraic equations whose roots are all pseudonegative (that is, with negative real parts). In Routh's work, for instance, such an equation $F(z)=0$ is expressed in the form $F(z)=E(z)+O(z)=A\left(z^{2}\right)+z B\left(z^{2}\right)$ where $E(z)$ is an even function of $z$ and $O(z)$ is an odd function of $z$ and it is found that the roots of the equations $A(x)=0, B(x)=0$ are all negative and occur alternately. The converse of this theorem has already been considered in section 2.2.

Foster also considers the driving-point impedance of a general network and connects it with a theorem associated with three positive definite quadratic forms which may be generalized as follows:

100 O. J. Zobel, Theory and design of uniform and composite electric wave-filters, Bell System Technical Journal vol. 2 (1923) pp. 35-36.

101 R. M. Foster, A reactance theorem, ibid. vol. 3 (1924) pp. 259-267; Theorems regarding the driving-point impedance of two-mesh circuits, ibid. vol. 3 (1924) pp. 651685. 
Consider the system of linear algebraic equations

$$
u_{r}=\sum_{s=1}^{n}\left(g_{r, s}+\lambda h_{r, s}+\lambda^{2} k_{r, s}\right) W_{s}, \quad r=1,2, \cdots, n,
$$

in which the coefficients $g_{r, s}, h_{r, s}, k_{r, s}$ are real symmetrical quantities such that $g_{r, s}=g_{s, r}, h_{r, s}=h_{s, r}, k_{r, s}=k_{s, r}$ and that $(x g x),(x h x),(x k x)$ are positive for any real nonvanishing set of quantities $x$. Then the zeros and poles of the function $w(\lambda)=\sum_{r=1}^{n} u_{r} W_{r}$ are all pseudo-negative.

When $\lambda$ is a complex quantity $\sigma+i \tau$ and $W_{r}=U_{r}+i V_{r}$, where $\sigma, \tau$, $U_{r}$ and $V_{r}$ are all real, the conjugate complex quantity to $W_{r}$, namely $W_{r}^{*}=U_{r}-i V_{r}$, arises when $\lambda$ is replaced by $\lambda^{*}=\sigma-i t$ and the zeros of $w(\lambda)$ will be pseudo-negative when the zeros of

$$
w^{*}(\lambda)=\sum_{r=1}^{n} u_{r} W_{r}^{*}=\left(W^{*} g W\right)+\lambda\left(W^{*} h W\right)+\lambda^{2}\left(W^{*} k W\right)
$$

are all pseudo-negative. When, however, the right-hand side is resolved into its real and imaginary parts and each of these equated to zero it is seen at once that $\sigma$ must be negative and a similar argument is applicable when $u=0$ and the possible values of $\lambda$ are, perhaps, poles of $w(\lambda)$. The theorem may be extended to the zeros and poles of the function $w(\lambda)+P$ where $P$ is a positive constant and may also be extended to certain integral equations of type

$$
u(s)=\int_{0}^{c}\left[g(s, t)+\lambda h(s, t)+\lambda^{2} k(s, t)\right] W(t) d t
$$

where $g(s, t), h(s, t), k(s, t)$ are real symmetric functions such that $(x g x),(x h x),(x k x)$ are positive for any nonvanishing function $x(s)$ continuous in the range $0 \leqq s \leqq c$.

Proofs and extensions of the reactance theorem have been given by Cauer, ${ }^{102}$ Baerwald, ${ }^{103}$ Epheser and Glubrecht ${ }^{104}$ and many other writ-

${ }^{102}$ W. Cauer, Die Verwirklichung von Wechselstrom Widerstanden vorgeschrieben Frequenzabhängigkeit, Dissertation, Technische Hochschule, Berlin, 1926, Arkiv för Elektrot. vol. 17 (1926) pp. 355-388; Ein Reaktanztheorem, Sitzungsbericht der Akademie der Wissenschaften, Berlin, 1931, pp. 673-681; Ein Satz über zwei zusammenhangen Hurwitzsche Polynome, Sitzungbericht der Berlin Mathematische Gesellschaft vol. 27 (1928) pp. 25-31; Wechstromschaltingen, Akademie der Wissenschaften, Leipzig, 1941.

${ }^{103}$ H. G. Baerwald, Ein einfacher Beweis der Reaktanztheorems, Elektrische Nachrichten Technik vol. 7 (1930) pp. 331-332.

${ }^{104}$ H. Epheser and H. Glubrecht, Die Grundlagen der Siebschaltungstheorien, ibid. vol. 17 (1940) pp. 169-192. See also K. Franz, Eine Verallgemeinerung des Fosterschen Reaktanztheorems auf beliebige Impidanzen, Elektrische Nachrichten Technik vol. 20 (1943) pp. 113-115. 
ers. There are also extensions to the case in which the quadratic forms $(x g x),(x h x),(x k x)$ contain an infinite number of variables and to the case in which the range of integration 0 to $c$ is infinite in the last mentioned theorem. Extensions to the case of Hermitian forms are also to be considered.

4.8. The sorting of vibrations by means of filters. It is often important to separate desirable vibrations from the undesirable ones particularly in electric and hydraulic transmission lines, but the problem of separation is important in acoustical and mechanical systems. In acoustics sound waves of a particular frequency or range of frequencies may be needed for experimental work as in the determination of times of reverberation in an auditorium. The elimination of noise is an important requirement in some buildings and laboratories. The absorption of the vibrations produced by engines and heavy machinery provides many mechanical problems.

The filtering properties of a system are generally examined by first finding the behavior of the system in a sinusoidal type of vibration, the so-called steady state, but it is important to know also the somewhat different response of the system to transient disturbances. This second problem is generally much harder than the first. Transient oscillations in electric wave filters were, however, studied by John Carson and Otto Zobel ${ }^{105}$ in 1923. The building up of sinusoidal currents in loaded electric lines had been investigated by Carson ${ }^{106}$ in 1917 and by Clark ${ }^{107}$ and Kupfmüller ${ }^{108}$ in 1923. Carson's analysis is

${ }^{105} \mathrm{~J}$. R. Carson and O. J. Zobel, Transient oscillations in electric wave filters, Bell System Technical Journal vol. 2 (1923) pp. 1-52.

${ }^{106} \mathrm{~J}$. R. Carson, Theory and calculation of variable electrical systems, Physical Review (2) vol. 17 (1921) pp. 116-134; General expansion theorem for the transient oscillations of a connected system, ibid. vol. 10 (1917) pp. 217-225; Theory of the transient oscillations of electrical networks and transmission systems, Transactions of the American Institute of Electrical Engineers vol. 38 (1919) pp. 345-427, discussion by M. I. Pupin and A. H. Cowles, pp. 462-464. See also T. C. Fry, The solution of circuit problems, Physical Review (2) vol. 14 (1919) pp. 115-136; J. R. Carson, The building up of sinusoidal currents in long periodically loaded lines, Bell System Technical Journal vol. 3 (1924) pp. 558-566; F. Pollaczek, Theory of the switching-on process of multimesh artificial lines, Elektrishe Nachrichten Technik vol. 2 (1925) pp. 197-226.

${ }^{107}$ Alva B. Clark, Telephone transmission over very long cable circuits, Transactions of the American Institute of Electrical Engineers vol. 42 (1923) pp. 86-97, discussion by J. J. Pilliod.

${ }^{108} \mathrm{~K}$. Küpfmüller, Free oscillation, echo effect, and influence of temperature in telephony over long Pupinised cables, Telegraphen- und Fernsprech-Technik vol. 12 (1923) pp. 53-60; Über Beziehungen zwischen Frequenzcharakteristiken und Ausgleichs vorgängen in linearen Systemen, Elektrische Nachrichten Technik vol. 5 (1928) pp. $18-32,459$. 
based largely on the use of Fourier integrals. For the simpler problem of transients in mechanically loaded lines when use can be made of partial differential equations or of differential difference equations with constant coefficients there is a powerful method of influence functions which has been used effectively by Koppe, Havelock, Schrödinger, Pollaczek and the present writer. ${ }^{109}$ Effective use can also be made of the inverse Laplace transformation as suggested by the writer in $1910 .{ }^{110}$ In 1921 at the present writer's suggestion, ${ }^{111}$ this method was adopted by Carson for work in electrical theory in which the so-called Duhamel integrals and operational methods of Heaviside were being used.

\subsection{Transverse vibrations of an infinite light string uniformly} loaded at regular intervals. The advantages of the methods mentioned in the last section may be seen by considering the function

$$
x_{n}(t)=\int_{0}^{t} J_{2 n}[2 c(t-s)] f(s) d s
$$

in which the integrand of this Poisson-Duhamel integral involves the influence function for the infinite loaded line. When $n$ is a positive or negative integer it is readily seen that $x_{n}(t)$ satisfies the homogeneous equation

$$
x_{n}^{\prime \prime}(t)=c^{2}\left[x_{n+1}(t)+x_{n-1}(t)-2 x_{n}(t)\right]
$$

but when $n=0$ it satisfies the nonhomogeneous equation

$$
x_{0}^{\prime \prime}(t)=f^{\prime}(t)+c^{2}\left(x_{1}+x_{-1}-2 x_{0}\right)
$$

${ }^{109}$ Some references to the literature are given in a paper by the author, Some simple differential difference equations and the related functions, Bull. Amer. Math. Soc. vol. 49 (1943) pp. 494-512.

${ }^{110} \mathrm{H}$. Bateman, The solution of a system of differential equations occurring in the theory of radio-active transformations, Proc. Cambridge Philos. Soc. vol. 15 (1910) pp. 423-427; The solution of linear differential equations by means of definite integrals, Trans. Cambridge Philos. Soc. vol. 21 (1909) pp. 171-196; Report on the history and present state of the theory of integral equations, Report of the British Association for the Advancement of Science, 1911, 80 pp.; An integral equation occurring in a mathematical theory of retail trade, Messenger of Mathematics vol. 49 (1919-1920) pp. 134-137.

${ }^{111}$ See a letter written by the author to J. R. Carson after the appearance of the latter's paper in Physical Review (1921). The method of the inverse Laplace transformation was recommended also by the author to M. D. Hersey in answer to a letter written during the last war regarding some equations occurring in the theory of recording instruments. The method has been taught by the writer for nearly forty years. Simeon Denis Poisson was probably the first man to use the method for the treatment of differential difference equations in his memoir Sur la distribution de la Chaleur dans les corps solides, J. Ecole Polytech. (1) vol. 12 (1923) pp. 1-144, 249-403 (especially pp. 29-34). 
and so the motion of the particle numbered 0 is forced while the motion of the other particles is free.

Following the lead of A. E. Heins ${ }^{112}$ who has used the method of the inverse Laplace transformation for the classical equation we form (A) the function

$$
X_{n}(z)=\int_{0}^{\infty} e^{-z t} x_{n}(t) d t
$$

and a difference equation for $X_{n}(z)$ can be readily found. In the particular case when $f(t)=\sin (2 a t)$ the multiplication theorem for integrals of Laplace's type gives the relation

$$
X_{n}(z)=2 a\left(z^{2}+4 a^{2}\right)^{-1}\left(z^{2}+4 c^{2}\right)^{-1 / 2}[T(z)]^{|2 n|}
$$

where

$$
2 c T(z)=\left(z^{2}+4 c^{2}\right)^{1 / 2}-z .
$$

From this expression for $X_{n}(z)$ the behavior of $x_{n}(t)$ for large positive values of $t$ may be derived by the method of Mellin and Haar explained in my paper in this Bulletin. Except for a damped initial motion the motion is eventually the steady state of periodic oscillation given by

$$
x_{n}(t) \sim\left(a^{2}-c^{2}\right)^{-1 / 2}\left[e^{2 i a t}\{T(2 i a)\}^{2 n} \pm e^{-2 i a t}\{T(-2 i a)\}^{2 n}\right] .
$$

When $a>c$ the right-hand side is real when the + sign is used, when $a<c$ the negative sign must be taken to make the right-hand side real. Another interesting example is that in which the pth particle differs in mass from all the others so that

$$
\begin{array}{lr}
x_{n}^{\prime \prime}=c^{2}\left(x_{n+1}+x_{n-1}-2 x_{n}\right), & n \neq p, \\
x_{p}^{\prime \prime}=a^{2}\left(x_{p+1}+x_{p-1}-2 x_{p}\right), & a \neq c, p>0 .
\end{array}
$$

A solution for the case in which $x_{0}(0)=1, x_{n}(0)=0, n \neq 0, x_{n}^{\prime}(0)=0$ is and

$$
x_{n}(t)=J_{2 n}(2 c t)+\left(1-c^{2} / a^{2}\right) \int_{0}^{t} J_{2 n-2 p}[2 c(t-s)] x_{p}^{\prime}(s) d s
$$

$$
X_{p}(z)=\frac{\{T(z)\}|2 p|}{\left(z^{2}+4 c^{2}\right)^{1 / 2}-\left(1-c^{2} / a^{2}\right) z} .
$$

112 A. E. Heins, On the solution of linear difference differential equations, Journal of Mathematics and Physics, Massachusetts Institute of Technology, vol. 19 (1940) pp. 153-157. 
The resulting estimate of $x_{p}(t)$ for large positive values of $t$ is for $c^{2}>2 a^{2}$

$$
x_{p}(t) \sim \frac{a^{2}\left(c^{2}-a^{2}\right)}{c^{2}\left(c^{2}-2 a^{2}\right)}\left(1-2 a^{2} / c^{2}\right)^{-|p|} \exp \left[-\frac{2 a^{2} t}{\left(c^{2}-2 a^{2}\right)^{1 / 2}}\right] .
$$

When $c^{2}>2 a^{2}$ the motion ultimately decays according to an exponential law. When $c^{2}<2 a^{2}$ the function $X_{p}(z)$ is infinite for two imaginary values of $z$ and $x_{p}(t)$ is ultimately periodic. When $c^{2}=2 a^{2}$ the solution is simply

$$
x_{p}(t)=(1 / 2 c t) J_{|2 p|+1}(2 c t) .
$$

California Institute of Technology 